\title{
Admissibility via Natural Dualities
}

\author{
Leonardo Cabrer ${ }^{\mathrm{a}}$, George Metcalfe ${ }^{\mathrm{a}, *}$ \\ ${ }^{a}$ Mathematics Institute, University of Bern \\ Sidlerstrasse 5, 3012 Bern, Switzerland
}

\begin{abstract}
It is shown that admissible clauses and quasi-identities of quasivarieties generated by a single finite algebra, or equivalently, the quasiequational and universal theories of their free algebras on countably infinitely many generators, may be characterized using natural dualities. In particular, axiomatizations are obtained for the admissible clauses and quasi-identities of bounded distributive lattices, Stone algebras, Kleene algebras and lattices, and De Morgan algebras and lattices.
\end{abstract}

Keywords: admissibility, natural dualities, quasivarieties, free algebras, De Morgan algebras 2000 MSC: Primary: 08C15, 08C20, 06D30, 08B20. Secondary: 06D50, 03C05, 03C13

\section{Introduction}

The concept of admissibility was introduced explicitly by Lorenzen in the 1950s in the context of intuitionistic logic [1], but played an important role already in the earlier work of Gentzen [2], Johansson [3], and Whitman [4]. Informally, a rule, consisting of a finite set of premises and a conclusion, is admissible for a system if adding it to the system produces no new theorems. Equivalently, the rule is admissible if for each of its instances, whenever the premises are theorems, the conclusion is also a theorem. Typically, proving that a rule is admissible for a system establishes that the system, or corresponding class of structures, has a certain property. For example, proving the admissibility of a "cut" rule for a proof system is a standard proof-theoretic strategy for establishing the completeness of the system for a given semantics (see, e.g. [2, 5]). Similarly, proving the admissibility of a "density" rule for a proof system has been used to establish that certain classes of algebraic structures are generated as varieties by their dense linearly ordered members (see, e.g. $[6,5]$ ). More generally, the addition of admissible rules to a system may be used to shorten proofs or speed up proof search.

In classical propositional logic, admissibility and derivability coincide; that is, the logic is structurally complete. However, this is not the case for non-classical logics such as intuitionistic logic and certain modal, many-valued, and substructural logics which formalize reasoning about, e.g., necessity and knowledge, vagueness and probability, and bounded resources and relevance. In particular, Rybakov proved in [7] (see also the monograph [8]) that the set of admissible rules of intuitionistic propositional logic is decidable but not finitely axiomatizable. Axiomatizations (called bases) of the admissible rules for this logic were later provided, independently, by Iemhoff [9] and Rozière [10], and subsequently for other intermediate logics by Iemhoff [11] and Cintula and Metcalfe [12], transitive modal logics by Jeřábek [13], temporal logics by Babyneshev and Rybakov [14, 15], and Łukasiewicz many-valued logics by Jeřábek [16, 17]. Bases are also given in these works for admissible multiple-conclusion rules where admissibility means that for each instance of the rule, whenever the premises are theorems, one of the conclusions is a theorem.

An algebraizable logic - roughly speaking, a logic with well-behaved translations between formulas and identities - is associated with a class of algebras called the equivalent algebraic

\footnotetext{
* Corresponding author

Email addresses: Imcabrer@yahoo.com.ar (Leonardo Cabrer), george.metcalfe@math.unibe.ch (George Metcalfe)
} 
semantics of the logic. This class is usually a quasivariety, in which case, rules correspond to quasiidentities and multiple-conclusion rules correspond to clauses (implications between conjunctions of identities and disjunctions of identities). Admissibility then amounts to the validity of quasiidentities or clauses in the free algebra on countably infinitely many generators of the quasivariety. Hence the study of admissibility may be viewed algebraically as the study of quasiequational and universal theories of free algebras. In particular, a crucial role is played here by subalgebras of (powers of) the free algebra on countably infinitely many generators. Indeed, in certain cases these algebras may be described and investigated using dualities. Notably, characterizations of admissible rules for intermediate and modal logics have been obtained using Kripke frames [18, $19,9,11,13,12]$, and for infinite-valued Lukasiewicz logic using rational polyhedra [16, 17].

For quasivarieties generated by a single finite algebra (e.g., Boolean algebras, distributive lattices, Stone algebras, Kleene algebras, De Morgan algebras), an accommodating and powerful dual framework may be provided by the theory of natural dualities (see [20]). In this setting, each algebra in the quasivariety is represented as an algebra of continuous morphisms over some structured Stone space, and there exists a dual equivalence between the quasivariety and the corresponding category of structured Stone spaces. Crucially for the approach described here, in the presence of a natural duality, free algebras of the quasivariety have a convenient representation on the dual side. The main goal of this paper is to show how natural dualities can be used to obtain bases for the admissible clauses and quasi-identities of quasivarieties generated by a finite algebra, or equivalently, axiomatizations of the quasiequational and universal theories of their free algebras on countably infinitely many generators. More precisely, it is shown that these bases can be derived from characterizations of the finite subalgebras of (finite powers of) the free algebras on countably infinitely many generators. These characterizations are obtained in turn via a combinatorial description of the dual objects of the algebras. Note that algorithms for checking admissibility of quasi-identities (but not clauses) in finitely generated quasivarieties have been obtained algebraically in $[21,22]$ but general methods for characterizing and axiomatizing admissible quasi-identities and clauses has until now been lacking.

As case studies, bases are obtained for the admissible quasi-identities and clauses of bounded distributive lattices, Stone algebras, De Morgan algebras and lattices, and Kleene algebras and lattices. Not only do these classes of algebras possess natural dualities already available in the literature [20], they have also been proposed as suitable semantics for applications in computer science. For example, De Morgan algebras (also called distributive $i$-lattices or quasi-Boolean algebras) have been used as suitable models for reasoning in databases (containing, e.g., extra elements representing "both true and false" and "neither true nor false") and as the basis for fuzzy logic systems (see, e.g., $[23,24]$ ). Some of these classes of algebras can be tackled without natural dualities (in particular, admissibility in bounded distributive lattices and Stone algebras admits a straightforward algebraic treatment). However, for other cases, such as Kleene and De Morgan algebras, the natural dualities play a crucial role in rendering the task feasible. A central goal of this paper is to emphasize the uniform approach to tackling these problems.

Following some preliminaries in Section 2 on universal algebra and natural dualities, Section 3 develops the necessary tools for characterizing admissible clauses and quasi-identities, focussing on locally finite quasivarieties. This approach is illustrated first in Section 4 with the relatively straightforward cases of (bounded) distributive lattices and Stone algebras. Sections 5 and 6 then treat, respectively, the more technically demanding cases of De Morgan algebras and lattices and Kleene algebras and lattices.

\section{Preliminaries}

Let us begin by recalling some basic material on universal algebra and natural dualities that will be needed in later sections. The reader is referred to [25] for background and undefined notions in category theory and to [26] and [20] for detailed explanations, definitions, and proofs concerning universal algebra and natural dualities, respectively. 


\subsection{Universal Algebra}

For convenience, let us assume in subsequent discussions that $\mathcal{L}$ is a finite algebraic language and that an $\mathcal{L}$-algebra $\mathbf{A}$ is an algebraic structure for this language, called trivial if $|A|=1$. We denote the formula algebra (absolutely free algebra) for $\mathcal{L}$ over countably infinitely many variables (free generators) by $\mathbf{F m}_{\mathcal{L}}$ and let the metavariables $\varphi, \psi, \chi$ stand for members of $\mathrm{Fm}_{\mathcal{L}}$ called $\mathcal{L}$-formulas. An $\mathcal{L}$-identity is an ordered pair of $\mathcal{L}$-formulas, written $\varphi \approx \psi$, and we let the metavariables $\Sigma, \Delta$ stand for finite sets of $\mathcal{L}$-identities. We also use $\varphi \preccurlyeq \psi$ as an abbreviation for $\varphi \wedge \psi \approx \varphi$ in the presence of the lattice meet operation.

An $\mathcal{L}$-clause is an ordered pair of finite sets of $\mathcal{L}$-identities, written $\Sigma \Rightarrow \Delta$. An $\mathcal{L}$-clause $\Sigma \Rightarrow \Delta$ is an $\mathcal{L}$-quasi-identity if $|\Delta|=1$, an $\mathcal{L}$-positive clause if $\Sigma=\emptyset$, and an $\mathcal{L}$-negative clause if $\Delta=\emptyset$. If both $|\Delta|=1$ and $\Sigma=\emptyset$, then the $\mathcal{L}$-clause is identified with the single $\mathcal{L}$-identity in $\Delta$.

Now let $\mathcal{K}$ be a class of $\mathcal{L}$-algebras and let $\Sigma \Rightarrow \Delta$ be an $\mathcal{L}$-clause. We write $\Sigma \models_{\mathcal{K}} \Delta$ to denote that for every $\mathbf{A} \in \mathcal{K}$ and homomorphism $h: \mathbf{F m}_{\mathcal{L}} \rightarrow \mathbf{A}$, whenever $\Sigma \subseteq \operatorname{ker} h$, then $\Delta \cap \operatorname{ker} h \neq \emptyset$. In this case, we say that each $\mathbf{A} \in \mathcal{K}$ satisfies $\Sigma \Rightarrow \Delta$ and that $\Sigma \Rightarrow \Delta$ is valid in $\mathcal{K}$. That is, $\Sigma \Rightarrow \Delta$ may be understood as the universal formula $(\forall \bar{x})(\bigwedge \Gamma \Rightarrow \bigvee \Delta)$ where $\bar{x}$ are the variables occurring in $\Gamma \cup \Delta$, and $\bigwedge \emptyset$ and $\bigvee \emptyset$ are identified with the truth constants 1 and 0 , respectively, of first-order logic. Conversely, an arbitrary universal formula of the language $\mathcal{L}$ may be associated with a finite set of $\mathcal{L}$-clauses, by putting the quantified formula into conjunctive normal form and interpreting each conjunct as an $\mathcal{L}$-clause.

We abbreviate $\emptyset \models_{\mathcal{K}} \Delta$ by $\models_{\mathcal{K}} \Delta$, and $\Sigma \models_{\{\mathbf{A}\}} \Delta$ by $\Sigma \models_{\mathbf{A}} \Delta$. We also drop the brackets in $\Sigma, \Delta$ when no confusion may occur. As usual, if the language is clear from the context we may omit the prefix $\mathcal{L}$ when referring to $\mathcal{L}$-algebras, $\mathcal{L}$-clauses, etc.

A set of $\mathcal{L}$-clauses $\Lambda$ is said to axiomatize $\mathcal{K}$ when $\mathbf{A} \in \mathcal{K}$ if and only if (henceforth "iff") all the clauses in $\Lambda$ are satisfied by $\mathbf{A}$. More generally, given another class $\mathcal{K}^{\prime}$ of $\mathcal{L}$-algebras, $\Lambda$ is said to axiomatize $\mathcal{K}$ relative to $\mathcal{K}^{\prime}$ when $\mathbf{A} \in \mathcal{K}$ iff both $\mathbf{A} \in \mathcal{K}^{\prime}$ and $\mathbf{A}$ satisfies all the clauses in $\Lambda$.

$\mathcal{K}$ is called an $\mathcal{L}$-universal class if there exists a set of $\mathcal{L}$-clauses $\Lambda$ that axiomatizes $\mathcal{K}$. If there exists such a $\Lambda$ consisting only of $\mathcal{L}$-identities, $\mathcal{L}$-quasi-identities, or $\mathcal{L}$-positive clauses, then $\mathcal{K}$ is called, respectively, an $\mathcal{L}$-variety, $\mathcal{L}$-quasivariety, or $\mathcal{L}$-positive universal class. The variety $\mathbb{V}(\mathcal{K})$, quasivariety $\mathbb{Q}(\mathcal{K})$, positive universal class $\mathbb{U}^{+}(\mathcal{K})$, and universal class $\mathbb{U}(\mathcal{K})$ generated by $\mathcal{K}$ are, respectively, the smallest $\mathcal{L}$-variety, $\mathcal{L}$-quasivariety, $\mathcal{L}$-positive universal class, and $\mathcal{L}$-universal class containing $\mathcal{K}$.

Let $\mathbb{H}, \mathbb{I}, \mathbb{S}, \mathbb{P}$, and $\mathbb{P}_{U}$ be, respectively, the class operators of taking homomorphic images, isomorphic images, subalgebras, products, and ultraproducts. Then

$$
\begin{aligned}
\mathbb{V}(\mathcal{K}) & =\mathbb{H} \mathbb{S P}(\mathcal{K}) & \mathbb{Q}(\mathcal{K}) & =\mathbb{I S P P}_{U}(\mathcal{K}) \\
\mathbb{U}^{+}(\mathcal{K}) & =\mathbb{H S P}_{U}(\mathcal{K}) & \mathbb{U}(\mathcal{K}) & =\operatorname{ISP}_{U}(\mathcal{K}) .
\end{aligned}
$$

Moreover, if $\mathcal{K}$ is a finite set of finite algebras, then

$$
\mathbb{Q}(\mathcal{K})=\mathbb{I S P}(\mathcal{K}) \quad \mathbb{U}^{+}(\mathcal{K})=\mathbb{H} \mathbb{S}(\mathcal{K}) \quad \mathbb{U}(\mathcal{K})=\mathbb{I S}(\mathcal{K}) .
$$

We refer to [26, Theorems II.9.5, II.11.9, V.2.20, and V.2.25] and [27, Exercise 3.2.2] for further details.

Let $\mathcal{K}$ be a class of $\mathcal{L}$-algebras and $\kappa$ a cardinal. An algebra $\mathbf{B} \in \mathcal{K}$ is called a free $\kappa$-generated algebra for $\mathcal{K}$ if there exists a set $X$ and a map $g: X \rightarrow \mathbf{B}$ such that $|X|=\kappa, g[X]$ generates $\mathbf{B}$, and for every $\mathbf{A} \in \mathcal{K}$ and map $f: X \rightarrow \mathbf{A}$ there exists a (unique) homomorphism $h: \mathbf{B} \rightarrow \mathbf{A}$ satisfying $f=h \circ g$. In this case, each $x \in X$ is called a free generator of $\mathbf{B}$.

For any quasivariety $\mathcal{Q}$, there exists for each cardinal $\kappa$, a unique (modulo isomorphism) free $\kappa$-generated algebra $\mathbf{F}_{\mathcal{Q}}(\kappa)$ in $\mathcal{Q}$. If $\mathcal{Q}$ contains at least one non-trivial algebra, then the map $g$ above is one-to-one and we may identify $X$ with the set $g[X]$. We denote the special case of the free $\omega$-generated algebra of $\mathcal{Q}$ by $\mathbf{F}_{\mathcal{Q}}$.

Let us recall some useful facts and properties. First, $\mathbf{F}_{\mathbb{V}(\mathcal{Q})}=\mathbf{F}_{\mathcal{Q}}$ (see [26, Corollary II.11.10]) and hence for any algebra $\mathbf{A}$, it follows that $\mathbf{F}_{\mathbb{V}(\mathbf{A})}=\mathbf{F}_{\mathbb{V}(\mathbb{Q}(\mathbf{A}))}=\mathbf{F}_{\mathbb{Q}(\mathbf{A})} \in \mathbb{Q}(\mathbf{A})$. Next note that identifying the free generators of $\mathbf{F} \mathbf{m}_{\mathcal{L}}$ with the free generators of $\mathbf{F}_{\mathcal{Q}}$, there exists a unique 
homomorphism $h_{\mathcal{Q}}: \mathbf{F m}_{\mathcal{L}} \rightarrow \mathbf{F}_{\mathcal{Q}}$ extending the identity map. We recall (see [26, Corollary II.11.6]) that, for each $\mathcal{L}$-identity $\varphi \approx \psi$,

$$
\models_{\mathcal{Q}} \varphi \approx \psi \quad \text { iff } \quad \models_{\mathbf{F}_{\mathcal{Q}}} \varphi \approx \psi \quad \text { iff } \quad h_{\mathcal{Q}}(\varphi)=h_{\mathcal{Q}}(\psi) .
$$

We note also that, since quasivarieties are closed under taking direct products, for any finite set of $\mathcal{L}$-identities $\Sigma \cup \Delta$,

$$
\Sigma \models_{\mathcal{Q}} \Delta \quad \text { iff } \quad \Sigma \models_{\mathcal{Q}} \varphi \approx \psi \text { for some } \varphi \approx \psi \in \Delta .
$$

Finally, it is helpful to observe that

$$
\models_{\mathcal{Q}} \Delta \quad \text { iff } \quad \models_{\mathcal{Q}} \varphi \approx \psi \text { for some } \varphi \approx \psi \in \Delta \quad \text { iff } \quad \models_{\mathbf{F}_{\mathcal{Q}}} \Delta .
$$

It only remains to check here that $\models_{\mathbf{F}_{\mathcal{Q}}} \Delta$ implies $\models_{\mathcal{Q}} \Delta$ as the other directions follow from (II) and $\mathbf{F}_{\mathcal{Q}} \in \mathcal{Q}$. Because $\Delta$ is finite, it involves only $n$ variables for some $n \in \mathbb{N}$, and hence $\models_{\mathcal{Q}} \Delta$ iff $\models_{\mathbf{A}} \Delta$ for each $n$-generated algebra $\mathbf{A}$ in $\mathcal{Q}$. But each such algebra is a homomorphic image of $\mathbf{F}_{\mathcal{Q}}$. So if $\models_{\mathbf{F}_{\mathcal{Q}}} \Delta$, then, since homomorphisms preserve positive universal clauses, also $\models_{\mathcal{Q}} \Delta$.

\subsection{Natural Dualities}

A structured topological space is a topological space $(X, \tau)$ together with a (possibly empty) finite set of relations defined on $X$ and a finite (possibly empty) set of internal operations on $X$. The general theory of natural dualities allows partial operations; however, the operations involved in the dualities considered in this paper will all be total.

Let us fix for the rest of this section a finite algebra $\mathbf{A}$ and a structured topological space $\underset{\sim}{A}$ with the same universe $A$ such that the topology on $\underset{\sim}{A}$ is the discrete topology. Let $\mathcal{A}$ denote the category whose objects are the algebras in $\mathbb{I S P}(\mathbf{A})$ and whose morphisms are homomorphisms of algebras. Let $\mathcal{X}$ be the class of all isomorphic copies of topologically closed substructures of direct powers (over non-empty index sets) of $\underset{\sim}{A}$. It is easy to see that $\mathcal{X}$ with continuous maps preserving the relational and functional structure spaces forms a category.

The structured topological space $\underset{\sim}{A}$ is said to be algebraic over $\mathbf{A}$ if the relations and the graphs of the operations in $\underset{\sim}{A}$ are contained in $\operatorname{SP}(\mathbf{A})$. In this case, contravariant functors $\mathrm{D}_{\mathcal{A}}: \mathcal{A} \rightarrow \mathcal{X}$ and $\mathrm{E}_{\mathcal{A}}: \mathcal{X} \rightarrow \mathcal{A}$ may be defined as described below. First, let

$$
\mathrm{D}_{\mathcal{A}}(\mathbf{B})=\mathcal{A}[\mathbf{B}, \mathbf{A}] \leq A_{\sim}^{B},
$$

where $\mathcal{A}[\mathbf{B}, \mathbf{A}]$ is the set of homomorphisms in $\mathcal{A}$ from $\mathbf{B}$ to $\mathbf{A}$ considered as a substructure and a topological subspace of $A_{\sim}^{B}$, and let $\mathrm{D}_{\mathcal{A}}(h: \mathbf{B} \rightarrow \mathbf{C})$ be the map $\mathrm{D}_{\mathcal{A}}(h): \mathrm{D}_{\mathcal{A}}(\mathbf{C}) \rightarrow \mathrm{D}_{\mathcal{A}}(\mathbf{B})$ defined by

$$
\mathrm{D}_{\mathcal{A}}(h)(f)=f \circ h \text { for each } f \in \mathrm{D}_{\mathcal{A}}(\mathbf{C}) .
$$

Observe that if $\mathbf{B}$ is finite, then $\mathrm{D}_{\mathcal{A}}(\mathbf{B})$ is finite and carries the discrete topology. Similarly, let

$$
\mathrm{E}_{\mathcal{A}}(X)=\mathcal{X}[X, A] \leq \mathbf{A}^{X},
$$

where $\mathcal{X}[X, A]$ is the set of morphisms from $X$ to $\underset{\sim}{A}$ considered as a subalgebra of $\mathbf{A}^{X}$, and let $\mathrm{E}_{\mathcal{A}}(l: X \rightarrow Y)$ be the map $\mathrm{E}_{\mathcal{A}}(l): \mathrm{E}_{\mathcal{A}}(Y) \rightarrow \mathrm{E}_{\mathcal{A}}(X)$ defined by

$$
\mathrm{E}_{\mathcal{A}}(l)(f)=f \circ l \text { for each } f \in \mathrm{E}_{\mathcal{A}}(Y) .
$$

The evaluation map determines a natural transformation from the identity functor in $\mathcal{A}$ into $\mathrm{E}_{\mathcal{A}} \circ \mathrm{D}_{\mathcal{A}}$. That is, for each $\mathbf{B} \in \mathcal{A}$, the map $e_{\mathbf{B}}: \mathbf{B} \rightarrow \mathrm{E}_{\mathcal{A}}\left(\mathrm{D}_{\mathcal{A}}(\mathbf{B})\right)$ defined by

$$
e_{\mathbf{B}}(b)(f)=f(b) \text { for each } b \in \mathbf{B} \text { and } f \in \mathrm{D}_{\mathcal{A}}(\mathbf{B})
$$

is a homomorphism and the class $\left\{e_{\mathbf{B}}\right\}_{\mathbf{B} \in \mathcal{A}}$ is a natural transformation. 
If $e_{\mathbf{B}}$ is an isomorphism for each $\mathbf{B} \in \mathcal{A}$, then $\mathrm{D}_{\mathcal{A}}$ and $\mathrm{E}_{\mathcal{A}}$ determine a dual equivalence between $\mathcal{A}$ and the category $\mathrm{D}_{\mathcal{A}}(\mathcal{A})$. In this case we say that $\underset{\sim}{A}$ determines a natural duality on $\mathcal{A}$. It is clear from the above that $\mathcal{A}$ encodes all the information needed to reproduce the functors that determine the equivalence. If $\mathcal{X}$ coincides with the category of isomorphic images of $\mathrm{D}_{\mathcal{A}}(\mathcal{A})$, then we say that $\mathcal{A}_{\sim}$ yields a full natural duality on $\mathcal{A}$.

One useful byproduct of having a natural duality for a class of algebras is the following:

Theorem 1 ([20, Corollary 2.2.4]). If $\underset{\sim}{A}$ yields a natural duality on $\mathcal{A}=\mathbb{I S P}(\mathbf{A})$, then, for each cardinal $\kappa$, the algebra $\mathrm{E}_{\mathcal{A}}\left(A_{\sim}^{\kappa}\right)$ is free $\kappa$-generated on $\mathcal{A}$.

We remark that all the dualities considered in this paper are strong. However, rather than provide the technical definition of a strong duality here (see [20, Section 3.2]), we instead recall two properties of strong dualities relevant to this paper. Namely, every strong duality is full, and embeddings (surjections) in $\mathcal{A}$ correspond to surjections (embeddings) in $\mathcal{X}$.

For the reader unfamiliar with natural dualities, let us present the Stone duality between Boolean algebras and Stone spaces (zero-dimensional compact Hausdorff topological spaces) as the standard example of this approach. First, recall that the class of Boolean algebras $\mathcal{B}$ coincides with $\operatorname{ISP}(\mathbf{2})$, where $\mathbf{2}$ denotes the two-element Boolean algebra. Let 2 be the 2 -element Stone space $(\{0,1\}, \mathcal{T})$, where $\mathcal{T}$ is the discrete topology. Given a Stone space $X$, the characteristic map of each clopen subset is a continuous map from $X$ into $\underset{\sim}{2}$. It follows that each Stone space is isomorphic to a closed subspace of direct powers of $\underset{\sim}{2}$, and that the functors $\mathrm{D}_{\mathcal{B}}(\mathbf{B})=\mathcal{B}[\mathbf{B}, \mathbf{2}]$ and $\mathrm{E}_{\mathcal{B}}(X)=\mathcal{X}[X, 2]$ determine a strong natural duality between Boolean algebras and Stone spaces. Moreover, the correspondence between $\mathcal{B}[\mathbf{B}, \mathbf{2}]$ and the set of ultrafilters of $\mathbf{B}$ determines a natural equivalence between the functor $D_{\mathcal{B}}$ and the usual presentation of Stone duality.

\section{Admissibility}

In this section, we introduce the notions of admissibility, structural completeness, and universal completeness, and relate these to free algebras and the generation of varieties and positive universal classes. We then refine these relationships in the context of locally finite quasivarieties, obtaining criteria (used repeatedly in subsequent sections) for a set of clauses or quasi-identities to axiomatize admissible clauses or quasi-identities relative to a quasivariety. Finally, we explain how natural dualities may be used to obtain these axiomatizations.

\subsection{Admissible Clauses}

Let $\mathcal{Q}$ be an $\mathcal{L}$-quasivariety. An $\mathcal{L}$-clause $\Sigma \Rightarrow \Delta$ is admissible in $\mathcal{Q}$ if for every homomorphism $\sigma: \mathbf{F m}_{\mathcal{L}} \rightarrow \mathbf{F m}_{\mathcal{L}}:$

$$
\models_{\mathcal{Q}} \sigma\left(\varphi^{\prime}\right) \approx \sigma\left(\psi^{\prime}\right) \text { for all } \varphi^{\prime} \approx \psi^{\prime} \in \Sigma \quad \text { implies } \quad \models_{\mathcal{Q}} \sigma(\varphi) \approx \sigma(\psi) \text { for some } \varphi \approx \psi \in \Delta .
$$

In particular, a negative $\mathcal{L}$-clause $\Sigma \Rightarrow \emptyset$ is admissible in $\mathcal{Q}$ iff there is no homomorphism $\sigma: \mathbf{F m}_{\mathcal{L}} \rightarrow \mathbf{F m}_{\mathcal{L}}$ satisfying $\models_{\mathcal{Q}} \sigma\left(\varphi^{\prime}\right) \approx \sigma\left(\psi^{\prime}\right)$ for all $\varphi^{\prime} \approx \psi^{\prime} \in \Sigma$.

The next result relates admissibility in $\mathcal{Q}$ to validity in the free algebra $\mathbf{F}_{\mathcal{Q}}$.

Theorem 2. Let $\mathcal{Q}$ be an $\mathcal{L}$-quasivariety, let $\Sigma \Rightarrow \Delta$ be an $\mathcal{L}$-clause, and let $\mathcal{U}$ be the universal class axiomatized by $\{\Sigma \Rightarrow \Delta\}$ relative to $\mathcal{Q}$. Then the following are equivalent:

(i) $\Sigma \Rightarrow \Delta$ is admissible in $\mathcal{Q}$.

(ii) $\Sigma \models \mathbf{F}_{\mathcal{Q}} \Delta$.

(iii) $\mathbb{U}^{+}(\mathcal{Q})=\mathbb{U}^{+}(\mathcal{U})$

Moreover, if $|\Delta|=1$, then the following is equivalent to (i)-(iii):

(iv) $\mathbb{V}(\mathcal{Q})=\mathbb{V}(\mathcal{U})$. 
Proof. (i) $\Rightarrow$ (ii). Suppose that $\Sigma \Rightarrow \Delta$ is admissible in $\mathcal{Q}$. Consider any homomorphism $g: \mathbf{F m}_{\mathcal{L}} \rightarrow \mathbf{F}_{\mathcal{Q}}$ satisfying $\Sigma \subseteq$ ker $g$, noting that if no such homomorphism exists, then trivially $\Sigma \models_{\mathbf{F}_{\mathcal{Q}}} \Delta$. Let us fix $f$ to be a map sending each variable $x$ to a formula $\varphi$ such that $h_{\mathcal{Q}}(\varphi)=g(x)$, and let $\sigma$ be the unique homomorphism $\sigma: \mathbf{F m}_{\mathcal{L}} \rightarrow \mathbf{F m}_{\mathcal{L}}$ extending $f$. Then $h_{\mathcal{Q}} \circ \sigma=g$ and $\Sigma \subseteq \operatorname{ker}\left(h_{\mathcal{Q}} \circ \sigma\right)$. So for $\varphi^{\prime} \approx \psi^{\prime} \in \Sigma$, also $h_{\mathcal{Q}}\left(\sigma\left(\varphi^{\prime}\right)\right)=h_{\mathcal{Q}}\left(\sigma\left(\psi^{\prime}\right)\right)$ and, by (I) from Section 2, $\models_{\mathcal{Q}} \sigma\left(\varphi^{\prime}\right) \approx \sigma\left(\psi^{\prime}\right)$. Hence, by assumption, $\models_{\mathcal{Q}} \sigma(\varphi) \approx \sigma(\psi)$ for some $\varphi \approx \psi \in \Delta$. Again by (I), $g(\varphi)=h_{\mathcal{Q}}(\sigma(\varphi))=h_{\mathcal{Q}}(\sigma(\psi))=g(\psi)$ as required.

(ii) $\Rightarrow$ (iii). Suppose that $\Sigma \models_{\mathbf{F}_{\mathcal{Q}}} \Delta$. So $\mathbf{F}_{\mathcal{Q}} \in \mathcal{U}$ and $\mathbb{U}^{+}\left(\mathbf{F}_{\mathcal{Q}}\right) \subseteq \mathbb{U}^{+}(\mathcal{U})$. Clearly $\mathbb{U}^{+}(\mathcal{U}) \subseteq$ $\mathbb{U}^{+}(\mathcal{Q})$, since $\mathcal{U} \subseteq \mathcal{Q}$. Using (III) from Section $2, \mathbb{U}^{+}(\mathcal{Q})=\mathbb{U}^{+}\left(\mathbf{F}_{\mathcal{Q}}\right)$. Hence $\mathbb{U}^{+}(\mathcal{Q})=\mathbb{U}^{+}(\mathcal{U})$.

(iii) $\Rightarrow$ (i). Suppose that $\mathbb{U}^{+}(\mathcal{Q})=\mathbb{U}^{+}(\mathcal{U})$ and let $\sigma: \mathbf{F m}_{\mathcal{L}} \rightarrow \mathbf{F m}_{\mathcal{L}}$ be a homomorphism such that $\models_{\mathcal{Q}} \sigma\left(\varphi^{\prime}\right) \approx \sigma\left(\psi^{\prime}\right)$ for all $\varphi^{\prime} \approx \psi^{\prime} \in \Sigma$. Because $\Sigma \models_{\mathcal{U}} \Delta$, also $\sigma(\Sigma) \models_{\mathcal{U}} \sigma(\Delta)$. Hence, since $\mathcal{U} \subseteq \mathcal{Q}$, it follows that $\models_{\mathcal{U}} \sigma(\Delta)$. But $\mathbb{U}^{+}(\mathcal{Q})=\mathbb{U}^{+}(\mathcal{U})$, so $\models_{\mathcal{Q}} \sigma(\Delta)$. By (III) from Section 2, $\models_{\mathcal{Q}} \sigma(\varphi) \approx \sigma(\psi)$ for some $\varphi \approx \psi \in \Delta$ as required.

Note finally for (iv) that if $|\Delta|=1$, then $\mathcal{U}$ is a quasivariety and (iii) is equivalent to $\mathbb{V}(\mathcal{Q})=$ $\mathbb{U}^{+}(\mathcal{U})=\mathbb{V}(\mathcal{U})$.

Example 3. The following lattice clauses (Whitman's condition, meet semi-distributivity, and join semi-distributivity, respectively) are satisfied by all free lattices (see [4, 28]):

$$
\begin{aligned}
& x \wedge y \preccurlyeq z \vee w \Rightarrow x \wedge y \preccurlyeq z, x \wedge y \preccurlyeq w, x \preccurlyeq z \vee w, y \preccurlyeq z \vee w \\
& x \wedge y \approx x \wedge z \Rightarrow x \wedge y \approx x \wedge(y \vee z) \\
& x \vee y \approx x \vee z \Rightarrow x \vee y \approx x \vee(y \wedge z) .
\end{aligned}
$$

Hence these clauses are admissible in the variety of lattices.

If $\mathbb{Q}\left(\mathbf{F}_{\mathcal{Q}}\right)$ is axiomatized by a set of quasi-identities $\Lambda$ relative to $\mathcal{Q}$, then we call $\Lambda$ a basis for the admissible quasi-identities of $\mathcal{Q}$. Similarly, if $\mathbb{U}\left(\mathbf{F}_{\mathcal{Q}}\right)$ is axiomatized by a set of clauses $\Lambda$ relative to $\mathcal{Q}$, then we call $\Lambda$ a basis for the admissible clauses of $\mathcal{Q}$.

Example 4. The following quasi-identities define the quasivariety of torsion-free groups relative to groups (in a language with $\cdot,^{-1}$, and $\mathrm{e}$ ) and are satisfied by all free groups:

$$
\underbrace{x \cdot \ldots \cdot x}_{n} \approx \mathrm{e} \quad \Rightarrow \quad x \approx \mathrm{e} \quad n=2,3, \ldots
$$

Hence these quasi-identities are admissible in the variety of groups. In fact, by the fundamental theorem of finitely generated abelian groups, every finitely generated torsion-free abelian group is isomorphic to a free abelian group $\mathbf{Z}^{n}$ for some $n \in \mathbb{N}$ (see, e.g., [29]). These quasi-identities therefore provide a basis for the admissible clauses (and, similarly, the admissible quasi-identities) of the variety of abelian groups.

Interest in admissible rules was first stimulated largely by the phenomenon of admissible nonderivable rules in intuitionistic propositional logic. In particular, it was shown by Rybakov in [7] (see also the monograph [8]) that the set of admissible rules of this logic, corresponding to admissible quasi-identities of the variety of Heyting algebras, is decidable but has no finite basis. Iemhoff [9] and Rozière [10] later proved, independently, that an infinite basis is formed by the family of "Visser rules", expressed algebraically as the quasi-identities

$$
\top \approx\left(\bigwedge_{i=1}^{n}\left(y_{i} \rightarrow x_{i}\right) \rightarrow\left(y_{n+1} \vee y_{n+2}\right)\right) \vee z \Rightarrow \top \approx \bigvee_{j=1}^{n+2}\left(\bigwedge_{i=1}^{n}\left(y_{i} \rightarrow x_{i}\right) \rightarrow y_{j}\right) \vee z \quad n=2,3, \ldots
$$

A basis for the admissible clauses of Heyting algebras is provided by the Visser rules together with clauses expressing, respectively, the absence of trivial algebras and the disjunction property:

$$
\top \approx \perp \Rightarrow \emptyset \quad \text { and } \quad \top \approx x \vee y \Rightarrow \top \approx x, \top \approx y .
$$


The proof of Iemhoff (also her work on intermediate logics in [11]) makes use of a characterization of finitely generated projective Heyting algebras (retracts of finitely generated free Heyting algebras) obtained by Ghilardi [18] in the dual setting of Kripke frames. Similarly, Ghilardi's Kripke frame characterizations of finitely generated projective algebras for various transitive modal logics [19] were used by Jeřábek [13] to obtain bases of admissible rules for these logics. In this paper, we will see that dual characterizations of finitely generated projective algebras can also play a central role in characterizing admissibility for quasivarieties generated by finite algebras.

\subsection{Structural Completeness and Universal Completeness}

A quasivariety $\mathcal{Q}$ is structurally complete if a quasi-identity is admissible in $\mathcal{Q}$ exactly when it is valid in $\mathcal{Q}$ : that is, $\Sigma \Rightarrow \varphi \approx \psi$ is admissible in $\mathcal{Q}$ iff $\Sigma \models_{\mathcal{Q}} \varphi \approx \psi$. Equivalent characterizations (first proved by Bergman in [30]) are obtained using Theorem 2 as follows.

Proposition 5 ([30, Proposition 2.3]). The following are equivalent for any $\mathcal{L}$-quasivariety $\mathcal{Q}$ :

(i) $\mathcal{Q}$ is structurally complete.

(ii) $\mathcal{Q}=\mathbb{Q}\left(\mathbf{F}_{\mathcal{Q}}\right)$.

(iii) For each quasivariety $\mathcal{Q}^{\prime} \subseteq \mathcal{Q}: \mathbb{V}\left(\mathcal{Q}^{\prime}\right)=\mathbb{V}(\mathcal{Q})$ implies $\mathcal{Q}^{\prime}=\mathcal{Q}$.

Proof. (i) $\Leftrightarrow$ (ii). Immediate from Theorem 2 .

(ii) $\Rightarrow$ (iii). Suppose that $\mathcal{Q}=\mathbb{Q}\left(\mathbf{F}_{\mathcal{Q}}\right)$ and let $\mathcal{Q}^{\prime} \subseteq \mathcal{Q}$ be a quasivariety satisfying $\mathbb{V}\left(\mathcal{Q}^{\prime}\right)=$ $\mathbb{V}(\mathcal{Q})$. Then $\mathbf{F}_{\mathcal{Q}^{\prime}}=\mathbf{F}_{\mathbb{V}\left(\mathcal{Q}^{\prime}\right)}=\mathbf{F}_{\mathbb{V}(\mathcal{Q})}=\mathbf{F}_{\mathcal{Q}}$, so $\mathcal{Q}=\mathbb{Q}\left(\mathbf{F}_{\mathcal{Q}}\right)=\mathbb{Q}\left(\mathbf{F}_{\mathcal{Q}^{\prime}} \subseteq \mathcal{Q}^{\prime}\right.$.

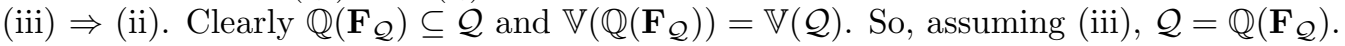

A quasivariety $\mathcal{Q}$ is universally complete if a clause is admissible in $\mathcal{Q}$ exactly when it is valid in $\mathcal{Q}$ : that is, $\Sigma \Rightarrow \Delta$ is admissible in $\mathcal{Q}$ iff $\Sigma \models_{\mathcal{Q}} \Delta$. We obtain the following characterization.

Proposition 6. The following are equivalent for any $\mathcal{L}$-quasivariety $\mathcal{Q}$ :

(i) $\mathcal{Q}$ is universally complete.

(ii) $\mathcal{Q}=\mathbb{U}\left(\mathbf{F}_{\mathcal{Q}}\right)$.

(iii) For each universal class $\mathcal{U} \subseteq \mathcal{Q}: \mathbb{U}^{+}(\mathcal{U})=\mathbb{U}^{+}(\mathcal{Q})$ implies $\mathcal{U}=\mathcal{Q}$.

Proof. (i) $\Leftrightarrow$ (ii). Immediate from Theorem 2 .

(i) $\Rightarrow$ (iii). Suppose that $\mathcal{Q}$ is universally complete and let $\mathcal{U} \subseteq \mathcal{Q}$ be a universal class with $\mathbb{U}^{+}(\mathcal{U})=\mathbb{U}^{+}(\mathcal{Q})$. Consider an $\mathcal{L}$-clause $\Sigma \Rightarrow \Delta$ such that $\Sigma \models_{\mathcal{U}} \Delta$ and let $\mathcal{U}^{\prime}$ be the universal class axiomatized relative to $\mathcal{Q}$ by $\{\Sigma \Rightarrow \Delta\}$. Then $\mathbb{U}^{+}(\mathcal{Q})=\mathbb{U}^{+}(\mathcal{U}) \subseteq \mathbb{U}^{+}\left(\mathcal{U}^{\prime}\right) \subseteq \mathbb{U}^{+}(\mathcal{Q})$. By Theorem $2, \Sigma \Rightarrow \Delta$ is admissible in $\mathcal{Q}$. As $\mathcal{Q}$ is universally complete, $\Sigma \models_{\mathcal{Q}} \Delta$. So $\Sigma \models_{\mathcal{U}} \Delta$ implies $\Sigma \models_{\mathcal{Q}} \Delta$, and hence $\mathcal{Q} \subseteq \mathcal{U}$.

(iii) $\Rightarrow$ (i). Suppose that $\Sigma \Rightarrow \Delta$ is admissible in $\mathcal{Q}$ and let $\mathcal{U}$ be the universal class axiomatized by $\{\Sigma \Rightarrow \Delta\}$ relative to $\mathcal{Q}$. By Theorem $2, \mathbb{U}^{+}(\mathcal{Q})=\mathbb{U}^{+}(\mathcal{U})$. So, assuming (iii), it follows that $\mathcal{U}=\mathcal{Q}$ and hence $\Sigma \models_{\mathcal{Q}} \Delta$.

Example 7. The variety of lattice-ordered abelian groups is, like every quasivariety, generated as a universal class by its finitely presented members. But every finitely presented lattice-ordered abelian group is a retract of a free finitely generated lattice-ordered abelian group (that is, a finitely generated projective lattice-ordered abelian group) [31]. Hence every finitely presented latticeordered abelian group embeds into the free lattice-ordered abelian group on countably infinitely many generators. So by Proposition 6, this variety is universally complete.

Certain classes of algebras are very close to being universally complete; in such classes, the lack of harmony between admissibility and validity is due entirely to trivial algebras, or, from a syntactic perspective, to non-negative clauses. An $\mathcal{L}$-quasivariety $\mathcal{Q}$ is non-negative universally complete if a non-negative $\mathcal{L}$-clause is admissible in $\mathcal{Q}$ exactly when it is valid in $\mathcal{Q}$ : that is, $\Sigma \Rightarrow\{\varphi \approx \psi\} \cup \Delta$ is admissible in $\mathcal{Q}$ iff $\Sigma \models_{\mathcal{Q}}\{\varphi \approx \psi\} \cup \Delta$. We obtain the following characterization: 
Proposition 8. The following are equivalent for any $\mathcal{L}$-quasivariety $\mathcal{Q}$ :

(i) $\mathcal{Q}$ is non-negative universally complete.

(ii) Every $\mathcal{L}$-clause admissible in $\mathcal{Q}$ is satisfied by all non-trivial members of $\mathcal{Q}$.

(iii) Every non-trivial algebra of $\mathcal{Q}$ is in $\mathbb{U}\left(\mathbf{F}_{\mathcal{Q}}\right)$.

Proof. (i) $\Rightarrow$ (ii). Suppose that $\mathcal{Q}$ is non-negative universally complete and let $\Sigma \Rightarrow \Delta$ be an $\mathcal{L}$-clause admissible in $\mathcal{Q}$. If $\Delta \neq \emptyset$, then, by assumption, $\Sigma \models_{\mathcal{Q}} \Delta$. Suppose now that $\Delta=\emptyset$ and let $x, y$ be variables not occurring in $\Sigma$. Then $\Sigma \Rightarrow x \approx y$ is admissible in $\mathcal{Q}$. Again, by assumption, $\Sigma \models_{\mathcal{Q}} x \approx y$. If $\mathbf{A} \in \mathcal{Q}$ is non-trivial, then $\Sigma \models_{\mathbf{A}} x \approx y$ iff $\Sigma \models_{\mathbf{A}} \emptyset$. So $\Sigma \Rightarrow \emptyset$ is satisfied by all non-trivial members of $\mathcal{Q}$.

(ii) $\Rightarrow$ (i). Follows immediately since any non-negative $\mathcal{L}$-clause is satisfied by all trivial $\mathcal{L}$ algebras.

(ii) $\Leftrightarrow$ (iii). By Theorem 2, an $\mathcal{L}$-clause is admissible in $\mathcal{Q}$ iff it is valid in $\mathbb{U}\left(\mathbf{F}_{\mathcal{Q}}\right)$, so (ii) is equivalent to the statement that $\mathbb{U}\left(\mathbf{F}_{\mathcal{Q}}\right)$ contains all non-trivial members of $\mathcal{Q}$, i.e., (iii).

Any non-negative universally complete $\mathcal{L}$-quasivariety $\mathcal{Q}$ is structurally complete. Moreover, if $\mathcal{Q}$ is not universally complete, then by Proposition 8 , any negative $\mathcal{L}$-clause not satisfied by trivial $\mathcal{L}$-algebras provides a basis for the admissible $\mathcal{L}$-clauses of $\mathcal{Q}$.

Example 9. Each non-trivial Boolean algebra, but no trivial Boolean algebra, embeds into a free Boolean algebra. Hence the variety of Boolean algebras is non-negative universally complete, but not universally complete, and $\perp \approx T \Rightarrow \emptyset$ forms a basis for its admissible clauses. The same holds for Boolean lattices (without $\perp$ and $\top$ in the language), but in this case, a suitable basis is formed by $x \wedge \neg x \approx x \vee \neg x \Rightarrow \emptyset$. In Section 4, we will see that bounded distributive lattices provide an example of a variety that is structurally complete, but neither universally complete nor non-negative universally complete.

\subsection{Local Finiteness}

The quasivarieties considered in subsequent sections of this paper are all locally finite: that is, their finitely generated members are finite. This property has important consequences for characterizations of the universal classes and quasivarieties generated by free algebras, which are in turn crucial for our study of admissible clauses and quasi-identities in these classes. In particular, we will make frequent use of the following result:

Lemma 10. For any locally finite $\mathcal{L}$-quasivariety $\mathcal{Q}$ :

(a) $\mathbf{A} \in \mathbb{U}\left(\mathbf{F}_{\mathcal{Q}}\right)$ iff each finite subalgebra $\mathbf{B}$ of $\mathbf{A}$ is in $\mathbb{I S}\left(\mathbf{F}_{\mathcal{Q}}\right)$.

(b) $\mathbf{A} \in \mathbb{Q}\left(\mathbf{F}_{\mathcal{Q}}\right)$ iff each finite subalgebra $\mathbf{B}$ of $\mathbf{A}$ is in $\operatorname{ISP}\left(\mathbf{F}_{\mathcal{Q}}\right)$.

Moreover, if $\mathcal{Q}=\mathbb{Q}(\mathcal{K})$ for some finite class $\mathcal{K}$ of finite $\mathcal{L}$-algebras, then

(c) $\mathbb{Q}\left(\mathbf{F}_{\mathcal{Q}}\right)=\mathbb{I S P}\left(\mathbf{F}_{\mathcal{Q}}\right)$.

Proof. First observe that, since universal classes are axiomatized by clauses, if $\mathcal{U}$ is a universal class and $\mathbf{A}$ is an algebra of the same language, then $\mathbf{A} \in \mathcal{U}$ iff each finitely generated subalgebra of $\mathbf{A}$ is in $\mathcal{U}$. Under the assumption of local finiteness, this is equivalent to each finite subalgebra of $\mathbf{A}$ being a member of $\mathcal{U}$. Hence, to establish (a) and (b), it suffices to prove:

(a') Each finite $\mathbf{B} \in \mathbb{U}\left(\mathbf{F}_{\mathcal{Q}}\right)$ is in $\mathbb{I S}\left(\mathbf{F}_{\mathcal{Q}}\right)$.

(b') Each finite $\mathbf{B} \in \mathbb{Q}\left(\mathbf{F}_{\mathcal{Q}}\right)$ is in $\operatorname{ISP}\left(\mathbf{F}_{\mathcal{Q}}\right)$. 
(a') Let $B=\left\{b_{1}, \ldots, b_{n}\right\}$ be an enumeration of the elements of $\mathbf{B} \in \mathbb{U}\left(\mathbf{F}_{\mathcal{Q}}\right)$. By assumption, there exists a non-empty set $I$, an ultrafilter $U$ over $I$, and an embedding $f: \mathbf{B} \rightarrow \mathbf{F}_{\mathcal{Q}}^{I} / U$. Let $\left\{g_{1}, \ldots, g_{n}\right\} \in \mathbf{F}_{\mathcal{Q}}^{I}$ be such that $f\left(b_{i}\right)=\left[g_{i}\right]_{U}$ for each $1 \leq i \leq n$. Because $f$ is one-to-one and $U$ is an ultrafilter of $I$, for each $1 \leq j<k \leq n$, the set $I_{j k}=\left\{i \in I \mid g_{j}(i) \neq g_{k}(i)\right\}$ is in $U$. Similarly, since $f$ is a homomorphism, for each $m$-ary operation $h$ of $\mathcal{L}$ and each $j_{1}, \ldots, j_{m} \in\{1, \ldots, n\}$, if $h\left(b_{j_{1}}, \ldots, b_{j_{m}}\right)=b_{k}$, the set $I_{h, j_{1}, \ldots, j_{m}}=\left\{i \in I \mid h\left(g_{j_{1}}(i), \ldots, g_{j_{m}}(i)\right)=g_{k}(i)\right\}$ is in $U$. Now, since $\mathcal{L}$ is finite,

$I^{\prime}=\bigcap\left\{I_{j k} \mid 1 \leq j<k \leq n\right\} \cap \bigcap\left\{I_{h, j_{1}, \ldots, j_{m}} \mid h\right.$ an $m$-ary operation of $\left.\mathcal{L}, 1 \leq j_{1}, \ldots, j_{m} \leq n\right\} \in U$.

So there exists $i \in I^{\prime}$ and it follows that the map $f^{\prime}: B \rightarrow \mathbf{F}_{\mathcal{Q}}$ defined by $f\left(b_{j}\right)=g_{j}(i)$ is a well-defined one-to-one homomorphism.

(b') Let B be a finite algebra in $\mathbb{Q}\left(\mathbf{F}_{\mathcal{Q}}\right)=\mathbb{I S P P}_{U}\left(\mathbf{F}_{\mathcal{Q}}\right)$. Then there exists a set $\mathcal{K}$ of algebras in $\mathbb{P}_{U}\left(\mathbf{F}_{\mathcal{Q}}\right)$ and an embedding $h: \mathbf{B} \rightarrow \prod \mathcal{K}$. For each $\mathbf{C} \in \mathcal{K}$ let $\pi_{\mathbf{C}}: \prod \mathcal{K} \rightarrow \mathbf{C}$ be the projection map. Then $\mathcal{K}^{\prime}=\left\{\pi_{\mathbf{C}} \circ h(\mathbf{B}) \mid \mathbf{C} \in \mathcal{K}\right\}$ satisfies $\mathbf{B} \in \mathbb{I S}\left(\prod \mathcal{K}^{\prime}\right)$ and $\mathcal{K}^{\prime} \subseteq \mathbb{S P}_{U}\left(\mathbf{F}_{\mathcal{Q}}\right)$. As $\mathbf{B}$ is finite, each member of $\mathcal{K}^{\prime}$ is finite. By (a'), $\mathcal{K}^{\prime} \subseteq \mathbb{I S}\left(\mathbf{F}_{\mathcal{Q}}\right)$. So $\mathbf{B} \in \mathbb{I S P S}\left(\mathbf{F}_{\mathcal{Q}}\right)=\mathbb{I S P}\left(\mathbf{F}_{\mathcal{Q}}\right)$.

(c) Suppose that $\mathcal{Q}=\mathbb{Q}(\mathcal{K})$ for some finite class $\mathcal{K}$ of finite $\mathcal{L}$-algebras and let $n$ be the maximum of the cardinalities of the members of $\mathcal{K}$. Then each member of $\mathcal{K}$ is a homomorphic image of $\mathbf{F}_{\mathcal{Q}}(n)$. So $\mathbb{V}\left(\mathbf{F}_{\mathcal{Q}}(n)\right)=\mathbb{V}(\mathcal{K})$ and hence $\mathbf{F}_{\mathcal{Q}} \in \mathbb{Q}\left(\mathbf{F}_{\mathcal{Q}}(n)\right)$ and $\mathbb{Q}\left(\mathbf{F}_{\mathcal{Q}}\right)=\mathbb{Q}\left(\mathbf{F}_{\mathcal{Q}}(n)\right)$. But since $\mathcal{K}$ is a finite class of finite algebras, $\mathbf{F}_{\mathcal{Q}}(n)$ is finite. So $\mathbb{Q}\left(\mathbf{F}_{\mathcal{Q}}\right)=\mathbb{Q}\left(\mathbf{F}_{\mathcal{Q}}(n)\right)=\operatorname{ISPP}_{U}\left(\mathbf{F}_{\mathcal{Q}}(n)\right)=$ $\mathbb{I S P}\left(\mathbf{F}_{\mathcal{Q}}(n)\right)=\mathbb{I S P}\left(\mathbf{F}_{\mathcal{Q}}\right)$.

Combining this result with Propositions 5 and 6 , we also obtain:

Corollary 11. For any locally finite $\mathcal{L}$-quasivariety $\mathcal{Q}$ :

(a) $\mathcal{Q}$ is universally complete iff each finite algebra in $\mathcal{Q}$ is in $\mathbb{I S}\left(\mathbf{F}_{\mathcal{Q}}\right)$.

(b) $\mathcal{Q}$ is structurally complete iff each finite algebra in $\mathcal{Q}$ is in $\operatorname{ISP}\left(\mathbf{F}_{\mathcal{Q}}\right)$.

Moreover, if $\mathcal{Q}=\mathbb{Q}(\mathcal{K})$ for some finite class $\mathcal{K}$ of finite $\mathcal{L}$-algebras, then

(c) $\mathcal{Q}$ is structurally complete iff $\mathcal{Q}=\operatorname{ISP}\left(\mathbf{F}_{\mathcal{Q}}\right)$.

To find a basis for the admissible clauses of a locally finite quasivariety $\mathcal{Q}$, it suffices, using Lemma 10, to find an axiomatic characterization of the finite subalgebras of $\mathbf{F}_{\mathcal{Q}}$. More precisely:

Lemma 12. Let $\mathcal{Q}$ be a locally finite $\mathcal{L}$-quasivariety.

(a) The following are equivalent for any set of $\mathcal{L}$-clauses $\Lambda$ :

(i) For each finite $\mathbf{B} \in \mathcal{Q}: \mathbf{B} \in \mathbb{I S}\left(\mathbf{F}_{\mathcal{Q}}\right)$ iff $\mathbf{B}$ satisfies $\Lambda$.

(ii) $\Lambda$ is a basis for the admissible clauses of $\mathcal{Q}$.

(b) The following are equivalent for any set of $\mathcal{L}$-quasi-identities $\Lambda$ :

(iii) For each finite $\mathbf{B} \in \mathcal{Q}: \mathbf{B} \in \mathbb{I S P}\left(\mathbf{F}_{\mathcal{Q}}\right)$ iff $\mathbf{B}$ satisfies $\Lambda$.

(iv) $\Lambda$ is a basis for the admissible quasi-identities of $\mathcal{Q}$.

Proof. (a) (i) $\Rightarrow$ (ii). It suffices to observe that for each $\mathbf{A} \in \mathcal{Q}$ :

A satisfies $\Lambda$ iff each finite subalgebra of $\mathbf{A}$ satisfies $\Lambda \quad(\mathcal{Q}$ is locally finite)

iff each finite subalgebra of $\mathbf{A}$ is in $\mathbb{I S}\left(\mathbf{F}_{\mathcal{Q}}\right)$ (assumption)

iff $\mathbf{A} \in \mathbb{U}\left(\mathbf{F}_{\mathcal{Q}}\right) \quad$ (Lemma 10(a)).

(ii) $\Rightarrow$ (i). Suppose that $\Lambda$ is a basis for the admissible clauses of $\mathcal{Q}$ and consider a finite $\mathbf{B} \in \mathcal{Q}$. By assumption, $\mathbf{B}$ satisfies $\Lambda$ iff $\mathbf{B} \in \mathbb{U}\left(\mathbf{F}_{\mathcal{Q}}\right)$. But then by Lemma $10(\mathrm{a}), \mathbf{B} \in \mathbb{U}\left(\mathbf{F}_{\mathcal{Q}}\right)$ iff $\mathbf{B} \in \mathbb{I S}\left(\mathbf{F}_{\mathcal{Q}}\right)$.

(b) Very similar to (a). 
We conclude this section by relating the problem of finding bases for the admissible clauses and quasi-identities of a quasivariety $\mathcal{Q}$ generated by a finite algebra $\mathbf{A}$ to natural dualities. Suppose that there is a structure $\underset{\sim}{A}$ that yields a strong natural duality on $\mathcal{Q}$. To obtain a basis for the admissible clauses of $\mathcal{Q}$, we seek a set of clauses $\Lambda$ that characterizes the finite algebras of $\mathcal{Q}$ that embed into $\mathbf{F}_{\mathcal{Q}}$. But these algebras correspond on the dual side to images of finite powers of $A$ under morphisms of the dual category. Hence we first seek conditions $C$ on dual spaces (in the cases considered in this paper, first-order conditions) to be images of finite powers of $\underset{\sim}{A}$ under morphisms of the dual category. We then seek a set of clauses $\Lambda$ such that a finite algebra $\mathbf{B} \in \mathcal{Q}$ satisfies the clauses in $\Lambda$ iff its dual space satisfies the conditions $C$. In this way, we avoid confronting the characterization of subalgebras of the free algebra $\mathbf{F}_{\mathcal{Q}}$ directly and consider rather the range of morphisms of the combinatorial structures $A_{\sim}^{n}$ for $n \in \mathbb{N}$. Similarly, to obtain a basis for the admissible quasi-identities of $\mathcal{Q}$, we seek a set of quasi-identities $\Lambda$ that characterizes the finite algebras of $\mathcal{Q}$ that embed into a finite power of $\mathbf{F}_{\mathcal{Q}}$, and therefore corresponding conditions on dual spaces to be images of finite copowers of finite powers of $\underset{\sim}{A}$ under morphisms of the dual category.

\section{Distributive Lattices and Stone Algebras}

In this section, we investigate and find bases for the admissible clauses and quasi-identities of (bounded) distributive lattices and Stone algebras. For the sake of uniformity and also as preparation for the more involved cases of Kleene and De Morgan algebras, we make use here of natural dualities. Let us remark, however, that in this setting direct algebraic proofs characterizing subalgebras of (powers of) free algebras are also relatively straightforward.

\subsection{Distributive Lattices}

The class of bounded distributive lattices $\mathcal{B D} \mathcal{L}$ is a variety and coincides with $\mathbb{I S P}(\mathbf{2})$, where $\mathbf{2}=(\{0,1\}$, min, $\max , 0,1)$ (see, e.g., $[26])$. Clearly $\mathbf{2} \in \mathbb{I S}\left(\mathbf{F}_{\mathcal{B D} \mathcal{L}}\right)$, so also $\mathcal{B D} \mathcal{L}=\mathbb{I} \mathbb{P P}(\mathbf{2}) \subseteq$ $\mathbb{Q}\left(\mathbf{F}_{\mathcal{B D} \mathcal{L}}\right)$ and, by Proposition 5:

Proposition 13. The variety of bounded distributive lattices is structurally complete.

However, $\mathcal{B D} \mathcal{L}$ is neither universally complete nor non-negative universally complete; that is, there are non-negative clauses that are admissible but not satisfied by all members of $\mathcal{B D} \mathcal{L}$. Here, we provide a basis for the admissible clauses of $\mathcal{B D} \mathcal{L}$, making use of the well-known natural duality between bounded distributive lattices and Priestley spaces (see [32] and [20, Theorem 4.3.2]).

Recall that a structured topological space $(X, \leq, \tau)$ is a Priestley space if $(X, \tau)$ is a compact topological space, $(X, \leq)$ is a partially ordered set, and for all $x, y \in X$ with $x \not \leq y$, there exists a clopen upset $U \subseteq X$ such that $x \in U$ and $y \notin U$. The structured topological space $\underset{\sim}{2}=\left(\{0,1\}, \leq_{2}, \mathcal{P}(\{0,1\})\right)$, where $\leq_{2}$ is the partial order on $\{0,1\}$ such that $0 \leq_{2} 1$, determines a strong natural duality between the variety $\mathcal{B D} \mathcal{L}$ and the category of Priestley spaces. In particular, by Theorem 1 applied to bounded distributive lattices, the free $n$-generated bounded distributive lattice is $\mathrm{E}_{\mathcal{B D} \mathcal{L}}\left(2_{\sim}^{n}\right)$ for $n \in \mathbb{N}$. Moreover, a finite bounded distributive lattice $\mathbf{L}$ is a retract of a free distributive lattice (that is, $\mathbf{L}$ is projective) iff the poset $\mathbf{D}_{\mathcal{B D} \mathcal{L}}(\mathbf{L})$ is a lattice (see [33]).

The following lemma provides the key step in our method for obtaining a basis for the admissible clauses of bounded distributive lattices (Theorem 15).

Lemma 14. Let $\mathbf{L}$ be a finite bounded distributive lattice. Then the following are equivalent:

(i) $\mathbf{L} \in \mathbb{I S}\left(\mathbf{F}_{\mathcal{B D} \mathcal{L}}\right)$.

(ii) $\mathrm{D}_{\mathcal{B D} \mathcal{L}}(\mathbf{L})$ is a non-empty bounded poset.

(iii) L satisfies the following clauses:

$$
\begin{gathered}
\top \approx \perp \Rightarrow \emptyset \\
x \wedge y \approx \perp \Rightarrow x \approx \perp, y \approx \perp \\
x \vee y \approx \top \Rightarrow x \approx \top, y \approx \top .
\end{gathered}
$$


Proof. (i) $\Rightarrow$ (ii). Since $\mathbf{L}$ is finite, $\mathbf{L} \in \mathbb{I S}\left(\mathbf{F}_{\mathcal{B D} \mathcal{L}}(n)\right)$ for some $n \in \mathbb{N}$. Let $h: \mathbf{L} \rightarrow \mathbf{F}_{\mathcal{B D} \mathcal{L}}(n)$ be a one-to-one homomorphism. 2 yields a strong duality, so $\mathrm{D}_{\mathcal{B D} \mathcal{L}}(h): \mathrm{D}_{\mathcal{B D L}}\left(\mathbf{F}_{\mathcal{B D} \mathcal{L}}(n)\right) \rightarrow \mathrm{D}_{\mathcal{B D} \mathcal{L}}(\mathbf{L})$ is an onto order-preserving map. But $\mathrm{D}_{\mathcal{B D} \mathcal{L}}\left(\mathbf{F}_{\mathcal{B D} \mathcal{L}}(n)\right)$ is isomorphic to $2_{\sim}^{n}$, so it is bounded, and hence $\mathrm{D}_{\mathcal{B D} \mathcal{L}}(\mathbf{L})$ is also bounded.

(ii) $\Rightarrow$ (i). Let us denote the top and bottom maps in $\mathrm{D}_{\mathcal{B D} \mathcal{L}}(\mathbf{L})=(X, \leq, \mathcal{P}(X))$ by $t$ and $s$, respectively. Let $\leq$ denote the partial order on $X$ defined as follows:

$$
x \leq^{\prime} y \quad \text { iff } \quad x=s \quad \text { or } \quad y=t \quad \text { or } \quad x=y .
$$

Clearly $\left(X, \leq^{\prime}\right)$ is a lattice. Hence $\mathrm{E}_{\mathcal{B D} \mathcal{L}}\left(X, \leq^{\prime}, \mathcal{P}(X)\right)$ is a retract of a finitely generated free distributive lattice, and it follows that $\mathrm{E}_{\mathcal{B D} \mathcal{L}}\left(X, \leq^{\prime}, \mathcal{P}(X)\right) \in \mathbb{I S}\left(\mathbf{F}_{\mathcal{B D} \mathcal{L}}\right)$.

Since $\leq^{\prime} \subseteq \leq$, the identity map $\operatorname{id}_{X}$ on $X$ is an order-preserving continuous map from $\left(X, \leq^{\prime}\right.$ $, \mathcal{P}(X))$ onto $\mathrm{D}_{\mathcal{B D} \mathcal{L}}(\mathbf{L})$. But 2 determines a strong natural duality, so the map $\mathrm{E}_{\mathcal{B D} \mathcal{L}}\left(\mathrm{id}_{X}\right) \circ$ $e_{\mathbf{L}}: \mathbf{L} \rightarrow \mathrm{E}_{\mathcal{B D} \mathcal{L}}\left(X, \leq^{\prime}, \mathcal{P}(X)\right)$ is one-to-one. Hence $\mathbf{L} \in \mathbb{I} S\left(\mathbf{F}_{\mathcal{B D} \mathcal{L}}\right)$.

(ii) $\Rightarrow$ (iii). Since $\mathrm{D}_{\mathcal{B D} \mathcal{L}}(\mathbf{L})$ is non-empty, $\mathbf{L}$ is non-trivial and satisfies (1). Suppose now that $s \in \mathrm{D}_{\mathcal{B D} \mathcal{L}}(\mathbf{L})$ is the bottom map of $\mathrm{D}_{\mathcal{B D} \mathcal{L}}(\mathbf{L})$, and that $c, d \in L$ are such that $c \vee d=\top$. Then $e_{\mathbf{L}}(c \vee d)(s)=e_{\mathbf{L}}(c)(s) \vee e_{\mathbf{L}}(d)(s)=1 \in \mathbf{2}$, so $e_{\mathbf{L}}(c)(s)=1$ or $e_{\mathbf{L}}(d)(s)=1$. Suppose that $e_{\mathbf{L}}(c)(s)=1$. Since $s$ is the bottom element of $\mathrm{D}_{\mathcal{B D} \mathcal{L}}(\mathbf{L})$ and $e_{\mathbf{L}}(c)$ is a monotone map, $e_{\mathbf{L}}(c)(z)=1$ for each $z \in \mathrm{D}_{\mathcal{B D} \mathcal{L}}(\mathbf{L})$; equivalently, $c=\top$. Similarly, $d=\top$ if $e_{\mathbf{L}}(d)(s)=1$. So $\mathbf{L}$ satisfies (3). Similarly, L satisfies (2) since $\mathrm{D}_{\mathcal{B D} \mathcal{L}}(\mathbf{L})$ has an upper bound.

(iii) $\Rightarrow$ (ii). By (3) and (1), the map $h$ defined by $h(\top)=1$ and $h(x)=0$ for each $x \neq \top$ is a homomorphism from $\mathbf{L}$ into $\mathbf{2}$ and is the bottom element of $\mathbf{D}_{\mathcal{B D} \mathcal{L}}(\mathbf{L})$. Similarly, using (2) and (1), the map $k$ defined by $k(\perp)=0$ and $k(x)=1$ if $x \neq \perp$ is the top element of $\mathrm{D}_{\mathcal{B D} \mathcal{L}}(\mathbf{L})$. So $\mathrm{D}_{\mathcal{B D} \mathcal{L}}(\mathbf{L})$ is a non-empty bounded poset.

Notice the key intermediary role played here by the natural duality in characterizing finite subalgebras of $\mathbf{F}_{\mathcal{B D L}}$ using the clauses (1), (2), and (3). On one hand, it is shown that satisfaction of the clauses by the finite algebra $\mathbf{L}$ corresponds to a certain characterization of $D_{\mathcal{B D} \mathcal{L}}(\mathbf{L})$. On the other hand, it is also shown that this characterization of $D_{\mathcal{B D} \mathcal{L}}(\mathbf{L})$ corresponds to $\mathbf{L} \in \mathbb{I S}\left(\mathbf{F}_{\mathcal{B D} \mathcal{L}}\right)$. Observe, moreover, that this second step makes use of the dual objects of a special subclass of $\mathbb{I S}\left(\mathbf{F}_{\mathcal{B D} \mathcal{L}}\right)$ : in this case, the finite projective distributive lattices. The structure of this proof will be repeated throughout the remainder of this paper.

Now, immediately, using Lemmas 12 and 14:

Theorem 15. $\{(1),(2),(3)\}$ is a basis for the admissible clauses of bounded distributive lattices.

By making a detour through the case of bounded distributive lattices, we can also show that the variety $\mathcal{D} \mathcal{L}$ of distributive lattices is universally complete.

Theorem 16. The variety of distributive lattices is universally complete.

Proof. First we describe a simple construction that will be employed with slight modifications in Sections 5.4 and 6.2. Given a distributive lattice $\mathbf{L}$, let $\overline{\mathbf{L}}$ denote the bounded distributive lattice obtained from $\mathbf{L}$ by adding fresh top and bottom elements $T, \perp$, respectively.

By Corollary 11(a), to establish the universal completeness of $\mathcal{D} \mathcal{L}$, it suffices to show that each finite distributive lattice $\mathbf{L}$ can be embedded into $\mathbf{F}_{\mathcal{D L}}$. Observe, however, that $\overline{\mathbf{L}}$ clearly satisfies the clauses (1), (2), and (3), so by Lemma 14, $\overline{\mathbf{L}} \in \mathbb{I S}\left(\mathbf{F}_{\mathcal{B D} \mathcal{L}}\right)$. But $\overline{\mathbf{F}}_{\mathcal{D L}} \cong \mathbf{F}_{\mathcal{B D} \mathcal{L}}$, so $\mathbf{L} \in \mathbb{I S}\left(\mathbf{F}_{\mathcal{D L}}\right)$ as required.

\subsection{Stone algebras}

An algebra $\left(A, \vee, \wedge,{ }^{*}, \perp, \top\right)$ is called a pseudocomplemented distributive lattice if $(A, \vee, \wedge, \perp, \top)$ is a bounded distributive lattice and $a^{*}=\max \{b \in A \mid a \wedge b=\perp\}$ for each $a \in A$. A pseudocomplemented distributive lattice is a Stone algebra if, additionally, $a^{*} \vee a^{* *}=\top$ for all $a \in A$. The class $\mathcal{S} \mathcal{T}$ of Stone algebras forms a variety whose unique proper non-trivial subvariety is the 


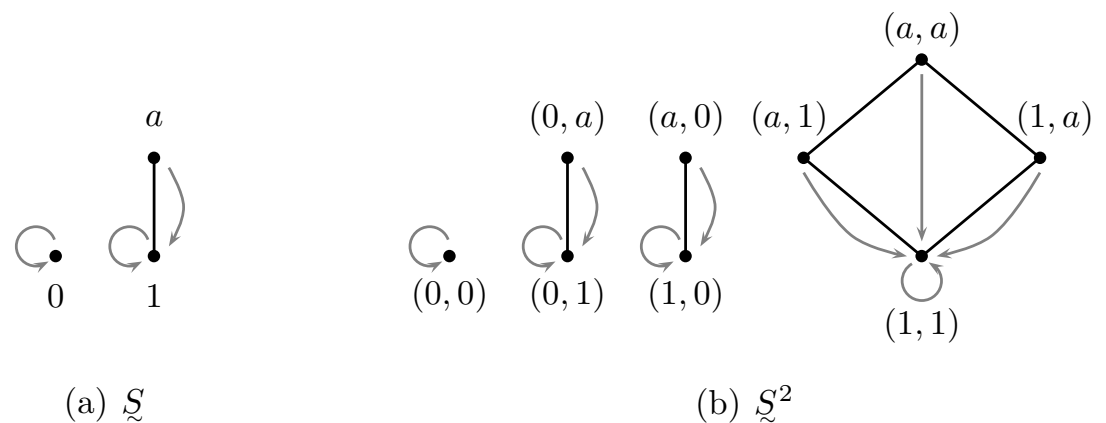

Figure 1: The spaces $\underset{\sim}{S}$ and $\underset{\sim}{S}$ ( $d$ is represented by arrows)

variety of Boolean algebras. We have already seen that the variety of Boolean algebras is nonnegative universally complete but not universally complete. In this section we will show that the same holds for $\mathcal{S T}$.

Let $\mathbf{S}=\left(\{0, a, 1\}, \wedge, \vee,^{*}, 0,1\right)$ denote the Stone algebra with order $0<a<1$ and $0^{*}=1$, $1^{*}=0$, and $a^{*}=0$. Then $\mathcal{S T}=\mathbb{I S P}(\mathbf{S})$ (see, e.g., [34]). Consider now the structure $\underset{\sim}{S}=$ $\left(\{0, a, 1\}, \leq_{S}, d, \mathcal{P}(\{0, a, 1\})\right)$ where $\leq_{S}$ is a partial order with a unique non-trivial edge $1 \leq_{S} a$ and $d:\{0, a, 1\} \rightarrow\{0, a, 1\}$ is defined by $d(a)=d(1)=1$ and $d(0)=0$ (see Figure $1($ a) $)$. Then $\underset{\sim}{S}$ determines a strong natural duality between $\mathcal{S} \mathcal{T}$ and the class of Priestley spaces endowed with a continuous map that sends each element $x$ to the unique minimal element $y$ satisfying $y \leq x$ (see [35] and [20, Theorem 4.3.7]). By Theorem 1, the dual space of the free $n$-generated Stone algebra $\mathrm{D}_{\mathcal{S T}}\left(\mathbf{F}_{\mathcal{S T}}(n)\right)$ is isomorphic to $S_{\sim}^{n}$ (Figure 1(b) depicts the case $\left.n=2\right)$.

Let us fix some notation. Given a poset $P$, let $\max (P)$ and $\min (P)$ denote the set of maximal and minimal elements of $P$, respectively. Trivially, if $P$ is finite, then $\max (P)$ and $\min (P)$ are non-empty.

Lemma 17. A finite Stone algebra belongs to $\mathbb{I S}\left(\mathbf{F}_{\mathcal{S T}}\right)$ if and only if it is non-trivial.

Proof. Since $\mathbf{F}_{\mathcal{S T}}$ is non-trivial, $T \neq \perp$ in $\mathbf{F}_{\mathcal{S T}}$ and each member of $\mathbb{I S}\left(\mathbf{F}_{\mathcal{S T}}\right)$ must contain at least two elements. This proves the left to right implication.

For the converse, first observe that $\max \left(\underset{\sim}{S}{ }^{n}\right)=\{0, a\}^{n}$. So $\max \left(\underset{\sim}{S}{ }^{n}\right)$ has $2^{n}$ elements and only $(0, \ldots, 0)$ among those elements satisfies $d(x)=x$. Let $\mathbf{A}$ be a finite non-trivial Stone algebra and let $\mathrm{D}_{\mathcal{S} \mathcal{T}}(\mathbf{A})=\left(\left\{x_{1}, \ldots, x_{k}\right\}, \leq, d, \mathcal{P}\left(\left\{x_{1}, \ldots, x_{k}\right\}\right)\right)$ be its dual space. Fix $n$ such that $k \leq 2^{n}-1$, and let $\left\{m_{1}, \ldots, m_{2^{n}}\right\}$ be an enumeration of the maximal elements of $S^{n}$, such that $m_{2^{n}}=(0, \ldots, 0)$. Fix $z \in \mathrm{D}_{\mathcal{S T}}(\mathbf{A})$ such that $d(z)=z$, and let $\eta:{\underset{\sim}{S}}^{n} \rightarrow \mathrm{D}_{\mathcal{S} \mathcal{T}}(\mathbf{A})$ be defined as follows:

$$
\eta(y)= \begin{cases}x_{i} & \text { if } y=m_{i} \text { for some } i \in\{1, \ldots, k\} \\ d\left(x_{i}\right) & \text { if } y<m_{i} \text { for some } i \in\{1, \ldots, k\} \\ z & \text { otherwise. }\end{cases}
$$

Then $\eta$ is a monotone onto map that preserves $d$. Hence $\mathrm{E}_{\mathcal{S T}}(\eta) \circ e_{\mathbf{A}}: \mathbf{A} \rightarrow \mathrm{E}_{\mathcal{S} \mathcal{T}}\left(\underset{\sim}{S^{n}}\right)$ is an embedding. Since $\mathbf{E}_{\mathcal{S T}}\left({\underset{\sim}{n}}^{n}\right)$ is isomorphic to $\mathbf{F}_{\mathcal{S T}}(n)$, the result follows.

We then obtain the following:

Theorem 18. The variety of Stone algebras is non-negative universally complete but not universally complete, and $\{(1)\}$ is a basis for the admissible clauses of this variety.

Proof. To prove that the variety $\mathcal{S} \mathcal{T}$ of Stone algebras is non-negative universally complete, it suffices, by Proposition 8, to check that every non-trivial Stone algebra $\mathbf{A}$ is in $\mathbb{U}\left(\mathbf{F}_{\mathcal{S T}}\right)$. This is true when $\mathbf{A}$ is finite by Lemma 17, and hence true in general by Lemma 10. $\mathcal{S T}$ fails to be 
universally complete since (1) is admissible but not satisfied by trivial Stone algebras, and this clause therefore provides a basis for the admissible clauses of the variety.

\section{De Morgan Algebras and Lattices}

A De Morgan lattice $(A, \wedge, \vee, \neg)$ is a distributive lattice equipped with an additional unary operation $\neg$ that is involutive $(a=\neg \neg a$ for all $a \in A)$ and satisfies the De Morgan law $\neg(a \wedge$ $b)=\neg a \vee \neg b$ for all $a, b \in A$. A De Morgan algebra $(A, \wedge, \vee, \neg, \perp, \top)$ consists of a bounded De Morgan lattice with additional constants $\perp$ and $T$ for the bottom and top elements of the lattice, respectively.

Our aim in this section is to provide bases for the admissible clauses and quasi-identities of the varieties $\mathcal{D} \mathcal{M A}$ of De Morgan algebras and $\mathcal{D} \mathcal{M L}$ of De Morgan lattices. More precisely, consider the following clauses, recalling that $\varphi \preccurlyeq \psi$ stands for $\varphi \wedge \psi \approx \varphi$ :

$$
\begin{aligned}
x \vee y \approx \top & \Rightarrow x \approx \top, y \approx \top \\
x \approx \neg x & \Rightarrow \emptyset \\
x \approx \neg x & \Rightarrow x \approx y \\
x \preccurlyeq \neg x, \neg(x \vee y) \preccurlyeq x \vee y, \neg y \vee z \approx \top & \Rightarrow z \approx \top \\
x \preccurlyeq \neg x, y \preccurlyeq \neg y, x \wedge y \approx \perp & \Rightarrow x \vee y \preccurlyeq \neg(x \vee y) .
\end{aligned}
$$

We prove the following:

- The clauses (3) and (4) form a basis for the admissible clauses of De Morgan algebras (Theorem 23).

- The quasi-identities (6) and (7) form a basis for the admissible quasi-identities of De Morgan algebras (Theorem 27).

- The clause (4) forms a basis for the admissible clauses of De Morgan lattices (Theorem 30).

- The quasi-identity (5) forms a basis for the admissible quasi-identities of De Morgan lattices (Theorem 30).

In [21] it is shown that (5) forms a basis for the admissible quasi-identities of De Morgan lattices, and that a quasi-identity is admissible in De Morgan algebras iff it is valid in all De Morgan algebras satisfying (3) and (5). This proof makes use of a description of the finite lattice of quasivarieties of De Morgan lattices provided by Pynko in [36]. However, it is the dual perspective on De Morgan algebras presented below that allows us to obtain a comprehensive description of admissibility in these varieties.

\subsection{A Natural Duality for De Morgan Algebras}

We obtain bases for the admissible clauses and quasi-identities of De Morgan algebras (and, with a little extra work, also for De Morgan lattices) by characterizing the dual spaces of the algebras in $\mathbb{I S}\left(\mathbf{F}_{\mathcal{D M \mathcal { A }}}\right)$ and $\mathbb{I S P}\left(\mathbf{F}_{\mathcal{D M \mathcal { A }}}\right)$ according to the following natural duality.

Consider first the De Morgan algebra $\mathbf{D}=(\{0, a, b, 1\}, \wedge, \vee, \neg, 0,1)$ with an order given by the Hasse diagram in Figure 2 (a) and $\neg 0=1, \neg 1=0, \neg a=a$, and $\neg b=b$. Then $\mathcal{D} \mathcal{M} \mathcal{A}=\mathbb{Q}(\mathbf{D})$ (see [37]). Now let $\underset{\sim}{D}=\left(\{0, a, b, 1\}, \leq_{D}, f, \mathcal{P}(\{0, a, b, 1\})\right)$ where $\leq_{D}$ is described by the Hasse diagram in Figure 2(b) and $f(0)=0, f(1)=1, f(a)=b$, and $f(b)=a$.

A structure $\left(X, \leq_{X}, f_{X}, \tau_{X}\right)$ is called a De Morgan space if

- $\left(X, \leq_{X}, \tau_{X}\right)$ is a Priestley space;

- $f_{X}$ is an involutive order-reversing homeomorphism. 


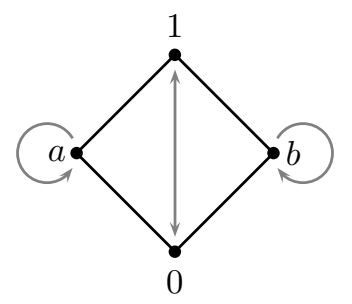

(a) $\mathbf{D}$

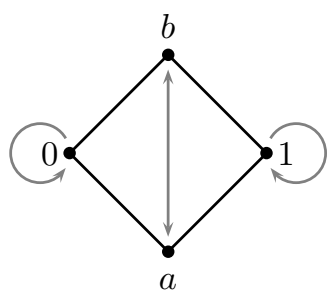

(b) $\underset{\sim}{D}$

Figure 2: The De Morgan algebra $\mathbf{D}$ and the space $\underset{\sim}{D}$

Theorem 19 ([20, Theorem 4.3.16]). The structure $\underset{\sim}{D}$ determines a strong natural duality between the variety of De Morgan algebras and the category of De Morgan spaces.

An application of Theorem 1 then establishes that for each $n \in \mathbb{N}$, the structure $\mathrm{E}_{\mathcal{D M \mathcal { A }}}\left(D^{n}\right)$ is the free $n$-generated De Morgan algebra.

The following lemma provides examples of (isomorphic copies of) subalgebras of $\mathbf{F}_{\mathcal{D M} \mathcal{A}}$ that will play an important role in the remainder of this section.

Lemma 20. Let $X$ be a finite set and $f$ an involution on $X$ with at least one fixpoint ( $x \in X$ with $f(x)=x)$. Consider $Y=\{u, v\} \cup X$ where $u \neq v$ and $u, v \notin X$, the map $\bar{f}: Y \rightarrow Y$ extending $f$ with $f(u)=v$ and $f(v)=u$, and the partial order $\leq$ on $Y$ defined by

$$
x \leq y \quad \Leftrightarrow \quad x=u \quad \text { or } \quad y=v \quad \text { or } \quad x=y .
$$

Then $\mathrm{E}_{\mathcal{D M A}}(Y, \leq, \bar{f}, \mathcal{P}(Y)) \in \mathbb{I S}\left(\mathbf{F}_{\mathcal{D M A}}\right)$.

Proof. Let $\left\{x_{0}, x_{1}, \ldots, x_{n}\right\}$ be an enumeration of the elements of $X$ such that $f\left(x_{0}\right)=x_{0}$. For each $i \in\{1, \ldots, n\}$, let $e_{i}=\left(\delta_{i 1}, \ldots, \delta_{i n}\right) \in D_{\sim}^{n}$ where $\delta_{i j}$ denotes the Kronecker delta; that is, $\delta_{i j}$ is 1 if $i=j$ and 0 otherwise.

Now we define $\eta: D^{n+2} \rightarrow Y$ as follows:

$$
\eta\left(z_{1}, \ldots, z_{n+2}\right)= \begin{cases}v & \text { if }\left|\left\{i \mid z_{i}=a\right\}\right|<\left|\left\{i \mid z_{i}=b\right\}\right| \\ u & \text { if }\left|\left\{i \mid z_{i}=a\right\}\right|>\left|\left\{i \mid z_{i}=b\right\}\right| \\ x_{i} & \text { if }\left(z_{1}, \ldots, z_{n+2}\right)=\left(e_{i}, a, b\right) \\ \bar{f}\left(x_{i}\right) & \text { if }\left(z_{1}, \ldots, z_{n+2}\right)=\left(e_{i}, b, a\right) \\ x_{0} & \text { otherwise. }\end{cases}
$$

It is a tedious but straightforward calculation to check that $\eta$ is a well-defined monotone map that preserves the involution.

Since $\eta$ is onto, by Theorem 19, $\mathrm{E}_{\mathcal{D M \mathcal { A }}}(\eta): \mathrm{E}_{\mathcal{D M \mathcal { A }}}(Y, \leq, \bar{f}, \mathcal{P}(Y)) \rightarrow \mathrm{E}_{\mathcal{D M \mathcal { A }}}\left(D_{\sim}^{n}\right)$ is a one-to-one homomorphism. The result then follows by Theorem 1 .

We remark that the algebra $\mathrm{E}_{\mathcal{D M A}}(Y, \leq, \bar{f}, \mathcal{P}(Y))$ defined in this lemma not only embeds into $\mathbf{F}_{\mathcal{D M A} \mathcal{A}}$, it is also a retract of $\mathbf{F}_{\mathcal{D M \mathcal { A }}}$. A characterization of finite retracts of free De Morgan algebras, the finite projective algebras of this variety, is given in [38].

\subsection{Admissible Clauses in De Morgan Algebras}

Our goal now is to describe the finite members of $\mathbb{I S}\left(\mathbf{F}_{\mathcal{D} \mathcal{M A}}\right)$ via a characterization of their counterparts in the dual space (Lemma 22), thereby obtaining a basis for the admissible clauses of $\mathcal{D} \mathcal{M A}$ (Theorem 23 ). We begin by proving two preparatory lemmas. 
Lemma 21. Let $\mathbf{A}$ be a finite De Morgan algebra and let $\mathrm{D}_{\mathcal{D M A} \mathcal{A}}(\mathbf{A})=(X, \leq, f, \tau)$ be its dual space. Then the following are equivalent:

(i) A satisfies (4).

(ii) There exists $x \in X$ such that $f(x)=x$.

Proof. (i) $\Rightarrow$ (ii). Suppose that $\mathbf{A}$ satisfies (4). Then $\mathbf{A}$ is non-trivial and $\mathcal{D} \mathcal{M A}[\mathbf{A}, \mathbf{D}]=X \neq \emptyset$. Assume now for a contradiction that $x \neq f(x)$ for each $x \in X$. We show that $\mathbf{A}$ must contain an element $c$ such that $\neg c=c$, contradicting (4). Let us define for each $x \in X$ :

$$
\begin{aligned}
C(x) & =\{C \subseteq X \mid C \text { is a chain and } \forall y \in C, y \leq x\} \\
m(x) & =\max \{|C| \mid C \in C(x)\} .
\end{aligned}
$$

We consider the sets:

$$
\begin{aligned}
U & =\{x \in X \mid m(x)<m(f(x))\} \\
V & =\{x \in X \mid m(f(x))<m(x)\} \\
W & =\{x \in X \mid m(x)=m(f(x))\} .
\end{aligned}
$$

Clearly $U, V$, and $W$ are pairwise disjoint and $X=U \cup V \cup W$. Moreover, $f(U)=V, f(V)=U$, and $f(W)=W$. Observe also that $U$ is a decreasing set. If $x \in U$ and $y \leq x$, then $f(x) \leq f(y)$, $C(y) \subseteq C(x)$, and $C(f(x)) \subseteq C(f(y))$. Thus $m(y) \leq m(x)<m(f(x)) \leq m(f(y))$ and $y \in U$. Also, if $x \in W$ and $y<x$, then $m(y)<m(x)=m(f(x))<m(f(y))$, so $y \in U$. A similar argument establishes that $V$ is an increasing set and that $W$ is an anti-chain.

Now let $W_{1}, W_{2} \subseteq W$ be a partition of $W$ such that $f\left(W_{1}\right)=W_{2}$. The existence of such a partition is ensured by the fact that $f(x) \neq x$ for each $x \in X$. Consider the map $\eta: X \rightarrow D \sim$ defined by:

$$
\eta(x)= \begin{cases}a & \text { if } x \in U \cup W_{1} \\ b & \text { if } x \in V \cup W_{2} .\end{cases}
$$

It follows that $\eta$ is well-defined and that $\neg \eta(x)=\eta(x)$ for each $x \in X$. Moreover, $\eta$ is increasing and preserves $f$. So $\eta \in \mathrm{E}_{\mathcal{D M \mathcal { A }}}\left(\mathrm{D}_{\mathcal{D M \mathcal { A }}}(\mathbf{A})\right)$. Let $c=e_{\mathbf{A}}^{-1}(\eta)$, so that $\neg c=c$.

(ii) $\Rightarrow$ (i). Suppose that there exists $h \in X=\mathcal{D} \mathcal{M} \mathcal{A}[\mathbf{A}, \mathbf{D}]$ such that $f(h)=h$. Now assume for a contradiction that there exists a homomorphism $g: \mathbf{F m}_{\mathcal{L}} \rightarrow \mathbf{A}$ satisfying $g(x)=g(\neg x)$. On one hand, $h(g(x))=f(h)(g(x))=f(h(g(x)))$. That is, $h(g(x))$ is a fixpoint of $f$ in $D$. Therefore, $h(g(x)) \in\{0,1\} \subseteq D$. On the other hand, $h(g(x))=h(g(\neg x))=\neg(h(g(x)))$ implies that $h(g(x))$

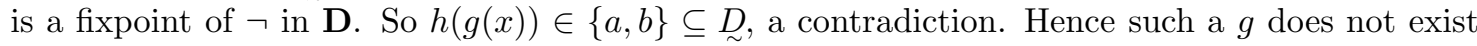
and (4) is satisfied.

Lemma 22. Let $\mathbf{A}$ be a finite De Morgan algebra and let $\mathrm{D}_{\mathcal{D M A} \mathcal{A}}(\mathbf{A})=(X, \leq, f, \tau)$ be its dual space. Then the following are equivalent:

(i) $\mathbf{A} \in \mathbb{I S}\left(\mathbf{F}_{\mathcal{D M A}}\right)$.

(ii) $(X, \leq)$ is non-empty and bounded and there exists $x \in X$ such that $f(x)=x$.

(iii) A satisfies (3) and (4).

Proof. (i) $\Rightarrow$ (ii). Suppose that $\mathbf{A} \in \mathbb{I S}\left(\mathbf{F}_{\mathcal{D M} \mathcal{A}}\right)$. Then, since $\mathbf{A}$ is finite, there exists $n \in\{1,2, \ldots\}$ and a one-to-one homomorphism $h: \mathbf{A} \rightarrow \mathbf{F}_{\mathcal{D M \mathcal { A }}}(n)$. So the map $\mathrm{D}_{\mathcal{D M \mathcal { A }}}(h): \mathrm{D}_{\mathcal{D M} \mathcal{A}}\left(\mathbf{F}_{\mathcal{D M} \mathcal{A}}(n)\right) \rightarrow$ $\mathrm{D}_{\mathcal{D M A} \mathcal{A}}(\mathbf{A})$ is onto. Since $\mathrm{D}_{\mathcal{D M \mathcal { A }}}(h)$ is order-preserving and $\mathrm{D}_{\mathcal{D M} \mathcal{A}}\left(\mathbf{F}_{\mathcal{D M} \mathcal{A}}(n)\right) \cong D_{\sim}^{n}$, it follows that $\mathrm{D}_{\mathcal{D M A}}(\mathbf{A})$ is bounded. Similarly, since $\mathrm{D}_{\mathcal{D M \mathcal { A }}}(h)$ preserves the involution and $\mathrm{D}_{\mathcal{D M \mathcal { A }}}\left(\mathbf{F}_{\mathcal{D M \mathcal { A }}}(n)\right)$ has $2^{n}$ fixpoints for the involution (every element in $\{0,1\}^{n}$ is a fixpoint in $\left.D^{n}\right), \mathbf{D}_{\mathcal{D M} \mathcal{A}}(\mathbf{A})$ has at least one fixpoint.

(ii) $\Rightarrow$ (i). Let us denote the top and bottom maps in $\mathrm{D}_{\mathcal{D} \mathcal{M} \mathcal{A}}(\mathbf{A})$ by $v$ and $u$, respectively. Let $\leq$ denote the partial order on $X$ defined as follows:

$$
x \leq^{\prime} y \quad \text { iff } \quad x=u \quad \text { or } \quad y=v \quad \text { or } \quad x=y .
$$


It follows from Lemma 20 that $\mathrm{E}_{\mathcal{D M} \mathcal{A}}\left(X, \leq^{\prime}, f, \mathcal{P}(X)\right) \in \mathbb{I S}\left(\mathbf{F}_{\mathcal{D M A} \mathcal{A}}\right)$.

Since $\leq^{\prime} \subseteq \leq$, the identity map $\operatorname{id}_{X}$ on $X$ is an order and involution-preserving map from $\left(X, \leq^{\prime}, f, \tau\right)$ onto $\mathrm{D}_{\mathcal{D M A} \mathcal{A}}(\mathbf{A})$. Hence, the map $\mathrm{E}_{\mathcal{D M A}}\left(\operatorname{id}_{X}\right) \circ e_{\mathbf{A}}: \mathbf{A} \rightarrow \mathrm{E}_{\mathcal{D M A}}\left(X, \leq^{\prime}, f, \tau\right)$ is oneto-one. So $\mathbf{A} \in \mathbb{I S}\left(\mathbf{F}_{\mathcal{D M \mathcal { A }}}\right)$.

(ii) $\Rightarrow$ (iii). By Lemma 21, A satisfies (4). To see that $\mathbf{A}$ satisfies (3), let $x$ be the bottom element and $f(x)$ the top element of $(X, \leq)$. Assume that $c, d \in \mathbf{A}$ are such that $c \vee d=\top$. Then the maps $e_{\mathbf{A}}(c)$ and $e_{\mathbf{A}}(d)$ satisfy $e_{\mathbf{A}}(c)(y) \vee e_{\mathbf{A}}(d)(y)=1$ for each $y \in X$. In particular, $e_{\mathbf{A}}(c)(x) \vee e_{\mathbf{A}}(d)(x)=1$. We claim that $e_{\mathbf{A}}(c)(x)=1$ or $e_{\mathbf{A}}(d)(x)=1$. Suppose for a contradiction that $e_{\mathbf{A}}(c)(x) \neq 1 \neq e_{\mathbf{A}}(d)(x)$. Then only two cases are possible: $e_{\mathbf{A}}(c)(x)=a$ and $e_{\mathbf{A}}(d)(x)=b$, or $e_{\mathbf{A}}(d)(x)=a$ and $e_{\mathbf{A}}(c)(x)=b$. If $e_{\mathbf{A}}(c)(x)=b$, then $e_{\mathbf{A}}(c)(f(x))=a$, but since $x$ is the bottom element of $(X, \leq)$, it follows that $x \leq f(x)$ and $e_{\mathbf{A}}(c)(x) \not \leq e_{\mathbf{A}}(c)(f(x))$ which contradicts the monotonicity of $e_{\mathbf{A}}$. A similar contradiction is obtained if we assume $e_{\mathbf{A}}(d)(x)=b$. Hence, without loss of generality, assume $e_{\mathbf{A}}(c)(x)=1$. Then $e_{\mathbf{A}}(c)(f(x))=1$. Since $e_{\mathbf{A}}(c)$ is a monotone map, for each $y \in X, 1=e_{\mathbf{A}}(c)(x) \leq e_{\mathbf{A}}(c)(y) \leq e_{\mathbf{A}}(c)(f(x))=1$. That is, $e_{\mathbf{A}}(c)$ is constantly 1 , establishing $c=\top$ as required.

(iii) $\Rightarrow$ (ii). If $\mathbf{A}$ satisfies (3) and (4), then $\top$ is join irreducible and $\perp$ is meet irreducible and $\perp \neq \top$ in $\mathbf{A}$. Hence, the map $h: \mathbf{A} \rightarrow \mathbf{D}$ defined by $h(\top)=1, h(\perp)=0$, and $h(x)=a$ if $x \notin\{\perp, \top\}$ is a homomorphism and therefore the bottom element of $\mathbf{D}_{\mathcal{D M} \mathcal{A}}(\mathbf{A})$. Clearly, $f(h)$ is the top element of $(X, \leq)$. So $(X, \leq)$ is non-empty and bounded. Also, by Lemma 21, there exists $x \in X$ such that $f(x)=x$.

Now immediately, by Lemmas 12 and 22:

Theorem 23. $\{(3),(4)\}$ is a basis for the admissible clauses of De Morgan algebras.

\subsection{Admissible Quasi-identities in De Morgan Algebras}

In order to present a basis for the admissible quasi-identities of De Morgan algebras we again need some preparatory lemmas.

Lemma 24. Let $\mathbf{A}$ be a finite De Morgan algebra and let $\mathrm{D}_{\mathcal{D M A} \mathcal{A}}(\mathbf{A})=(X, \leq, f, \tau)$ be its dual space. Then the following are equivalent:

(i) A satisfies (6).

(ii) For every $x \in \min (X, \leq)$, there exists $z \in X$ such that $x \leq z$ and $f(z)=z$.

Proof. (i) $\Rightarrow$ (ii). If $\mathbf{A}$ is trivial, then (ii) is clearly satisfied. Suppose then for a contradiction that $\mathbf{A}$ is non-trivial and that there exists $x \in \min (X, \leq)$ such that for all $z \geq x, f(z) \neq z$.

Let $Y=\{z \in X \mid f(z) \neq z\}$. Then $Y$ with the structure inherited from $\mathrm{D}_{\mathcal{D} \mathcal{M A}_{\mathcal{A}}}(\mathbf{A})$ is a nontrivial De Morgan space without fixpoints. In the proof of Lemma 21, it was shown that given such a De Morgan space $Y$ without fixpoints, there exists a map $\eta: Y \rightarrow D$ such that $\neg \eta=\eta$; that is, $\eta(Y) \subseteq\{a, b\}$.

Now, consider $\gamma, \mu, \nu: \mathbf{D}_{\mathcal{D M \mathcal { A }}}(\mathbf{A}) \rightarrow \underset{\sim}{D}$ defined as follows:

$\gamma(u)=\left\{\begin{array}{ll}\eta(u) & \text { if } u \neq f(u) \\ 0 & \text { if } u=f(u)\end{array} \quad \mu(u)=\left\{\begin{array}{ll}0 & \text { if } x \leq u \leq f(x) \\ b & \text { if } x<u \text { and } u \not \leq f(x) \\ a & \text { if } u<f(x) \text { and } x \not \leq u \\ 1 & \text { otherwise }\end{array} \quad \nu(u)= \begin{cases}a & \text { if } x=u \\ b & \text { if } f(x)=u \\ 1 & \text { otherwise. }\end{cases}\right.\right.$

It is an easy calculation to prove that $\gamma, \mu, \nu \in \mathrm{E}_{\mathcal{D M \mathcal { A }}}\left(\mathrm{D}_{\mathcal{D M \mathcal { A }}}(\mathbf{A})\right)$. Now observe that since $\gamma(X) \subseteq\{0, a, b\}$ and $(\gamma \vee \mu)(X) \in\{a, b, 1\}$, it follows that $\gamma \leq \neg \gamma$ and $\neg(\gamma \vee \mu) \leq \gamma \vee \mu$. Moreover, since $\nu(u) \neq 1$ iff $u \in\{x, f(x)\}$ and $\mu(\{x, f(x)\})=\{0\}, \neg \mu \vee \nu=1$ and $\nu \neq 1$, contradicting (6).

(ii) $\Rightarrow$ (i). Let $c, d, e \in A$ be such that $c \leq \neg c, \neg(c \vee d) \leq c \vee d$, and $\neg d \vee e=\top$. Consider $x \in \min (X, \leq)$. By hypothesis, there exists $y \in X$ such that $x \leq y$ and $f(y)=y$. Then 
$e_{\mathbf{A}}(c)(y)=e_{\mathbf{A}}(c)(f(y))$ and $e_{\mathbf{A}}(d)(y)=e_{\mathbf{A}}(d)(f(y))$. So $e_{\mathbf{A}}(c)(y), e_{\mathbf{A}}(d)(y) \in\{0,1\}$. Since $e_{\mathbf{A}}(c)(y) \leq \neg\left(e_{\mathbf{A}}(c)(y)\right)$, it follows that $e_{\mathbf{A}}(c)(y) \in\{0, a, b\}$ and therefore $e_{\mathbf{A}}(c)(y)=0$.

Thus $\neg\left(e_{\mathbf{A}}(c)(y) \vee e_{\mathbf{A}}(d)(y)\right)=\neg\left(e_{\mathbf{A}}(d)(y)\right) \leq e_{\mathbf{A}}(c)(y) \vee e_{\mathbf{A}}(d)(y)=e_{\mathbf{A}}(d)(y)$, which implies that $e_{\mathbf{A}}(d)(y)=1$ and therefore $\neg e_{\mathbf{A}}(d)(y)=0$. Then $\neg e_{\mathbf{A}}(d)(x) \in\{0, a\}$ and $e_{\mathbf{A}}(e)(y)=$ $e_{\mathbf{A}}(e)(f(y))$. Therefore, $e_{\mathbf{A}}(e)(y)=1$. On one hand, since $e_{\mathbf{A}}(e)(x) \leq e_{\mathbf{A}}(e)(y)=1$, it follows that $e_{\mathbf{A}}(e)(x) \in\{a, 1\}$. On the other hand, since $e_{\mathbf{A}}(e)(x) \vee \neg e_{\mathbf{A}}(d)(x)=1 \in \mathbf{D}$, it follows that $e_{\mathbf{A}}(e)(x) \in\{b, 1\}$. So $e_{\mathbf{A}}(e)(x)=1$. Since this can be proved for every minimal element of $(X, \leq)$ and every maximal element is equal to $f(x)$ for some minimal element, $e_{\mathbf{A}}(e)(x)=1$ for every minimal or maximal element $x$ of $(X, \leq)$. Since $e_{\mathbf{A}}(e)$ is increasing, we conclude that $e_{\mathbf{A}}(c)$ is constantly equal to 1 ; that is, $c=T$.

Lemma 25. Let $\mathbf{A}$ be a finite De Morgan algebra and let $\mathrm{D}_{\mathcal{D M A} \mathcal{A}}(\mathbf{A})=(X, \leq, f, \tau)$ be its dual space. Then the following are equivalent:

(i) A satisfies (7).

(ii) For every $x \in X$, there exists $y \in X$ such that $y \leq x, f(x)$.

Proof. (i) $\Rightarrow$ (ii). If $\mathbf{A}$ is trivial, then (ii) is clearly satisfied. Otherwise, suppose that $\mathbf{A}$ is non-trivial and that there exists $x \in \mathrm{D}_{\mathcal{D M \mathcal { A }}}(\mathbf{A})$ such that for all $y \in \mathrm{D}_{\mathcal{D} \mathcal{M} \mathcal{A}}(\mathbf{A})$, either $y \not \leq x$ or $y \not \leq f(x)$. Consider $\eta, \mu: \mathbf{D}_{\mathcal{D M A}}(\mathbf{A}) \rightarrow \underset{\sim}{D}$ defined as follows:

$$
\eta(u)=\left\{\begin{array}{ll}
a & \text { if } u \leq x \\
b & \text { if } u \geq f(x) \\
0 & \text { otherwise }
\end{array} \quad \mu(u)= \begin{cases}a & \text { if } u \leq f(x) \\
b & \text { if } u \geq x \\
0 & \text { otherwise }\end{cases}\right.
$$

If $u \leq x$ and $u \geq f(x)$, then $f(x) \leq x$, contradicting the assumption that, since $f(x) \leq f(x)$, $f(x) \not \leq x$. So $\eta$ is well-defined. Similarly, $\mu$ is well-defined.

It is easily observed that $\eta, \mu \in \mathrm{E}_{\mathcal{D M \mathcal { A }}}\left(\mathrm{D}_{\mathcal{D} \mathcal{M} \mathcal{A}}(\mathbf{A})\right)$ and that $\eta \leq \neg \eta, \mu \leq \neg \mu$. Letting $c=e_{\mathbf{A}}^{-1}(\eta)$ and $d=e_{\mathbf{A}}^{-1}(\mu)$, it follows that $c \leq \neg c$ and $d \leq \neg d$. Consider $u \in \mathrm{D}_{\mathcal{D M} \mathcal{A}}(\mathbf{A})$. If $\eta(u)=a$, then $u \leq x$ and, by assumption, $u \not \leq f(x)$. Thus $\mu(u) \in\{b, 0\}$, which implies $(\eta \wedge \mu)(u)=\eta(u) \wedge \mu(u)=0 \in \mathbf{D}$. Similarly, if $\eta(u)=b$, then $u \geq f(x)$ and $u \not \leq x$. Thus $\mu(u) \in\{a, 0\}$, and $(\eta \wedge \mu)(u)=0$. So $\eta \wedge \mu$ is constantly 0; that is, $c \wedge d=\perp$. But $(\eta \vee \mu)(x)=$ $\eta(x) \vee \mu(x)=1$, and therefore $(\eta \vee \mu)(x)=1 \not \leq \neg(\eta \vee \mu)(x)=0$; that is, $c \vee d \not \leq \neg(c \vee d)$ which contradicts (7).

(ii) $\Rightarrow$ (i). Consider $c, d \in A$ such that $c \leq \neg c, d \leq \neg d$, and $c \wedge d=\perp$. Let $x \in \mathrm{D}_{\mathcal{D M} \mathcal{A}}(\mathbf{A})$. Suppose now for a contradiction that $\left(e_{\mathbf{A}}(c) \vee e_{\mathbf{A}}(d)\right)(x)=1$. It follows from the assumptions that either $e_{\mathbf{A}}(c)(x)=a$ and $e_{\mathbf{A}}(d)(x)=b$, or $e_{\mathbf{A}}(c)(x)=b$ and $e_{\mathbf{A}}(d)(x)=a$. If $e_{\mathbf{A}}(c)(x)=a$ and $e_{\mathbf{A}}(d)(x)=b$, then there exists $y \in \mathrm{D}_{\mathcal{D} \mathcal{M} \mathcal{A}}(\mathbf{A})$ such that $y \leq x, f(x)$. But then $e_{\mathbf{A}}(c)(y) \leq$ $e_{\mathbf{A}}(c)(x)=a$ and $e_{\mathbf{A}}(d)(y) \leq e_{\mathbf{A}}(d)(f(x))=a$. So $e_{\mathbf{A}}(c)(y) \wedge e_{\mathbf{A}}(d)(y)=a \neq 0$, contradicting the assumption that $c \wedge d=\perp$. A similar contradiction is obtained if we assume $e_{\mathbf{A}}(c)(x)=b$ and $e_{\mathbf{A}}(d)(x)=a$.

We conclude that $\left(e_{\mathbf{A}}(c) \vee e_{\mathbf{A}}(d)\right)(x) \neq 1$ for each $x \in \mathrm{D}_{\mathcal{D} \mathcal{M} \mathcal{A}}(\mathbf{A})$; that is $e_{\mathbf{A}}(c \vee d)\left(\mathrm{D}_{\mathcal{D} \mathcal{M} \mathcal{A}}(\mathbf{A})\right) \subseteq$ $\{0, a, b\}$. Therefore $c \vee d \leq \neg(c \vee d)$.

Lemma 26. Let $\mathbf{A}$ be a finite non-trivial De Morgan algebra and let $\mathrm{D}_{\mathcal{D M A} \mathcal{A}}(\mathbf{A})=(X, \leq, f, \tau)$ be its dual space. Then the following are equivalent:

(i) $\mathbf{A} \in \mathbb{I S P}\left(\mathbf{F}_{\mathcal{D M A}}\right)$.

(ii) $(X, \leq, f, \tau)$ satisfies the following conditions:

(a) For every $x \in \min (X, \leq)$, there exists $z \in X$ such that $x \leq z=f(z)$.

(b) For every $x \in X$, there exists $y \in X$ such that $y \leq x, f(x)$.

(iii) A satisfies (6) and (7). 
Proof. (ii) $\Leftrightarrow$ (iii). Immediate from Lemmas 24 and 25 .

(i) $\Rightarrow$ (ii). Since $\mathbf{A}$ is finite, by (i), there exist $n, m \in\{1,2, \ldots\}$ and a one-to-one homomorphism $h: \mathbf{A} \rightarrow \mathbf{F}_{\mathcal{D M \mathcal { A }}}(n)^{m}$. So $\mathrm{D}_{\mathcal{D M} \mathcal{A}}(h): \coprod_{i=1}^{m} D^{n} \rightarrow \mathrm{D}_{\mathcal{D M} \mathcal{A}}(\mathbf{A})$ is an onto map.

Let $x \in X$ and $y \in \coprod_{i=1}^{m} D^{n}$ be such that $\mathrm{D}_{\mathcal{D M \mathcal { A }}}(h)(y)=x$. Note that $\coprod_{i=1}^{m} D_{\sim}^{n}$ is just the disjoint union of $m$ different copies $D_{\sim}^{n}$ of $D_{\sim}^{n}$. So there is an $i$ such that $y \in D_{\sim}^{n}$. Let $z=(a, \ldots, a) \in D_{i}^{n}$, then $\mathrm{D}_{\mathcal{D M A} \mathcal{A}}(h)(z) \leq x, f(x)$ which proves $(\mathrm{b})$.

If $x \in \min (X, \leq)$, then $\mathrm{D}_{\mathcal{D M} \mathcal{A}}(h)(z)=x$. Let $t=(0, \ldots, 0) \in D_{i}^{n}$, then $x \leq \mathrm{D}_{\mathcal{D M} \mathcal{A}}(h)(t)=$ $f\left(\mathrm{D}_{\mathcal{D M \mathcal { A }}}(h)(t)\right)$, and (a) follows.

(ii) $\Rightarrow$ (i). For each $x \in \min (X, \leq)$, let $[x, f(x)]=\{z \in X \mid x \leq z \leq f(x)\}$. By condition (a), there exists $z \in[x, f(x)]$ such that $f(z)=z$. Let us consider $[x, f(x)]$ endowed with the involution, poset, and topological structure inherited from $X$. Then $[x, f(x)]$ satisfies all the conditions of Lemma 22 (iii). Hence

$$
\mathrm{E}_{\mathcal{D M A}}([x, f(x)]) \in \mathbb{I} S\left(\mathbf{F}_{\mathcal{D M A}}\right) .
$$

By definition, trivially the inclusion map $\iota_{x}:[x, f(x)] \rightarrow X$ is monotone and preserves the involution. Consider $Y=\coprod_{x \in \min (X, \leq)}[x, f(x)]$ and $\iota: Y \rightarrow X$ the unique morphism such that $\iota_{[x, f(x)]}=\iota_{x}$. By (b), $\iota$ is an onto morphism in the category of Priestley spaces with an involution. Now Theorem 19 implies that the homomorphism $\mathrm{E}_{\mathcal{D M A}}(\iota): \mathrm{E}_{\mathcal{D M \mathcal { A }}}\left(\mathrm{D}_{\mathcal{D} \mathcal{M} \mathcal{A}}(\mathbf{A})\right) \rightarrow \mathrm{E}_{\mathcal{D M A}}(Y)$ is one-to-one. However,

$$
\mathrm{E}_{\mathcal{D M A}}(Y)=\mathrm{E}_{\mathcal{D M A} \mathcal{A}}\left(\coprod_{x \in \min (X, \leq)}[x, f(x)]\right) \cong \prod_{x \in \min (X, \leq)} \mathrm{E}_{\mathcal{D M \mathcal { A }}}([x, f(x)]) \in \mathbb{I} \mathbb{S P}\left(\mathbf{F}_{\mathcal{D M A}}\right) .
$$

Hence $\mathbf{A} \cong \mathrm{E}_{\mathcal{D M A}}\left(\mathrm{D}_{\mathcal{D} \mathcal{M} \mathcal{A}}(\mathbf{A})\right) \in \mathbb{I S P}\left(\mathbf{F}_{\mathcal{D} \mathcal{M A}}\right)$ as required.

Again, immediately by Lemmas 12 and 26:

Theorem 27. $\{(6),(7)\}$ is a basis for the admissible quasi-identities of De Morgan algebras.

\subsection{Admissibility in De Morgan Lattices}

Characterizations of admissibility in De Morgan lattices follow relatively straightforwardly from the above characterizations for De Morgan algebras. We first extend the definition of $\overline{\mathbf{L}}$ given in Section 4 to the case of De Morgan algebras and lattices. Let $\mathbf{A}=(A, \wedge, \vee, \neg)$ be a De Morgan lattice. Then $\overline{\mathbf{A}}=\left(A, \wedge, \vee, \neg^{\prime}, \top, \perp\right)$ denotes the De Morgan algebra that results from adding to the lattice $(A, \wedge, \vee)$ fresh top and bottom elements $T$ and $\perp$ with $\neg^{\prime}(\top)=\perp, \neg^{\prime}(\perp)=\top$, and $\neg^{\prime}(x)=\neg(x)$ otherwise. Note that $\overline{\mathbf{A}}$ satisfies (3).

Lemma 28. Let $\mathbf{A}$ be a finite De Morgan lattice. Then the following are equivalent:

(i) $\mathbf{A} \in \mathbb{I S}\left(\mathbf{F}_{\mathcal{D M} \mathcal{L}}\right)$.

(ii) A satisfies (4).

Proof. Observe first that $\mathbf{F}_{\mathcal{D M \mathcal { A }}}(\kappa) \cong \overline{\mathbf{F}}_{\mathcal{D M \mathcal { L }}}(\kappa)$. Hence, easily, each finite De Morgan lattice $\mathbf{A}$ is in $\mathbb{I S}\left(\mathbf{F}_{\mathcal{D M L}}\right)$ iff $\overline{\mathbf{A}} \in \mathbb{I S}\left(\mathbf{F}_{\mathcal{D M A}}\right)$. Moreover, by Lemma $22, \overline{\mathbf{A}} \in \mathbb{I S}\left(\mathbf{F}_{\mathcal{D M A}}\right)$ iff $\overline{\mathbf{A}}$ satisfies (3) and (4). Hence, since $\overline{\mathbf{A}}$ always satisfies (3), $\mathbf{A} \in \mathbb{I S}\left(\mathbf{F}_{\mathcal{D M L}}\right)$ iff $\overline{\mathbf{A}}$ satisfies (4). Since $\overline{\mathbf{A}}$ satisfies (4) iff A satisfies (4), the result follows.

Lemma 29. Let $\mathbf{A}$ be a finite De Morgan lattice. Then the following are equivalent:

(i) $\mathbf{A} \in \mathbb{I S P}\left(\mathbf{F}_{\mathcal{D M} \mathcal{L}}\right)$.

(ii) A satisfies (5).

Proof. The result follows from Lemma 28 and the fact that a De Morgan lattice $\mathbf{B}$ satisfies (5) iff it is a trivial algebra or satisfies (4).

So by Lemmas 12, 28, and 29:

Theorem 30. $\{(4)\}$ and $\{(5)\}$ are bases for the admissible clauses and admissible quasi-identities of De Morgan lattices, respectively. 


\section{Kleene Algebras and Lattices}

De Morgan algebras and lattices have only two non-trivial proper subvarieties apiece, Boolean algebras and lattices, and Kleene algebras and lattices (see [37]). As shown in Example 9, Boolean algebras and Boolean lattices are non-negative universally complete but not universally complete. However, the varieties of Kleene algebras and lattices both have admissible quasi-identities and clauses that are not derivable.

Recall that Kleene lattices and Kleene algebras are, respectively, De Morgan lattices and De Morgan algebras satisfying the Kleene condition $a \wedge \neg a \leq b \vee \neg b$ for all elements $a, b$. Consider now the clauses:

$$
\begin{aligned}
\top \approx \perp & \Rightarrow \emptyset \\
x \vee y \approx \top & \Rightarrow x \approx \top, y \approx \top \\
x \approx \neg x & \Rightarrow \emptyset \\
\neg x \preccurlyeq x, x \wedge \neg y \preccurlyeq \neg x \vee y & \Rightarrow \neg y \preccurlyeq y .
\end{aligned}
$$

In this section, we prove the following:

- The clauses (1), (3), and (8) form a basis for the admissible clauses of Kleene algebras (Theorem 35).

- The quasi-identity (8) forms a basis for both the admissible quasi-identities of Kleene algebras (Theorem 37) and the admissible quasi-identities of Kleene lattices (Theorem 40).

- The clauses (4) and (8) form a basis for the admissible clauses of Kleene lattices (Theorem 40).

In [21], an alternative proof is given of the fact that (8) forms a basis for the admissible quasiidentities of Kleene lattices and algebras. This proof makes use of a description of the finite lattice of quasivarieties of Kleene lattices provided by Pynko in [36]. However, as in the case of De Morgan algebras, a suitable natural duality provides a method for characterizing also the admissible clauses of these varieties.

\subsection{A Natural Duality for Kleene Algebras}

Let $\mathbf{K}=(\{0, a, 1\}, \wedge, \vee, \neg, 0,1)$ be the Kleene algebra where $0<a<1$ and $\neg 0=1, \neg 1=0$, and $\neg a=a$ (see Figure 3(a)). Then $\mathcal{K} \mathcal{A}=\mathbb{Q}(\mathbf{K})$ (see [37]). Let also $\underset{\sim}{K}=\left(\{0, a, 1\}, \leq_{K}, \sim_{K}\right.$ $,\{0,1\}, \mathcal{P}(\{0, a, 1\}))$ where $\leq_{K}$ is defined by $0,1 \leq_{K} a$ and 0,1 are incomparable (see Figure $3(\mathrm{~b})$ ), and $\sim_{K} \subseteq K^{2}$ is as depicted in Figure $3(\mathrm{c})$.

A structure $\left(X, \leq_{X}, \sim_{X}, Y_{X}, \tau_{X}\right)$ is called a Kleene space if

- $\left(X, \leq_{X}, \tau_{X}\right)$ is a Priestley space.

- $\sim_{X}$ is a closed binary relation; i.e., $\sim_{X}$ is a closed subset of $X^{2}$.

- $Y_{X}$ is a closed subset of $X$.

- For all $x, y, z \in X$ :

$-x \sim_{X} x$

- If $x \sim_{X} y$ and $x \in Y_{X}$, then $x \leq_{X} y$.

- If $x \sim_{X} y$ and $y \leq_{X} z$, then $z \sim_{X} x$.

Theorem 31 ([39]). K determines a strong natural duality between the variety of Kleene algebras and the category of Kleene spaces (see also [20, Theorem 4.3.10]).

The following lemma provides a subclass of $\mathbb{I S}\left(\mathbf{F}_{\mathcal{K} \mathcal{A}}\right)$ that will play a crucial role in the proof of Lemma 34 . 


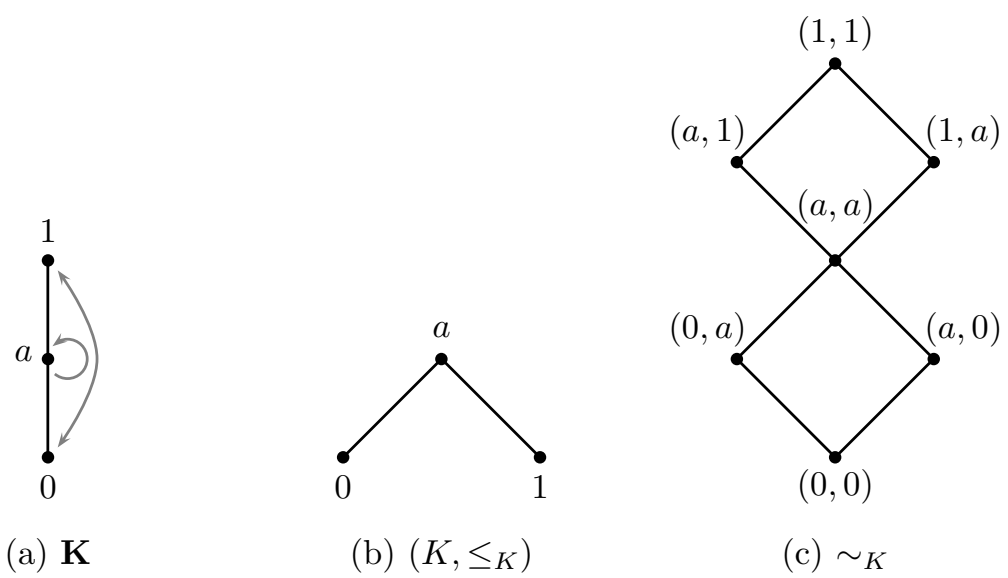

Figure 3: The Kleene algebra $\mathbf{K}$ and the Kleene space $\underset{\sim}{K}$

Lemma 32. Let $(X, \leq)$ be a finite tree; that is, $(X, \leq)$ is a poset with a top element such that for each $x, y \in X, z \leq x, y$ for some $z \in X$ iff $(x \leq y$ or $y \leq x)$. Let $\sim_{X}=\leq \cup \geq$ and $Y_{X}=\min (X, \leq)$. Then $\mathrm{E}_{\mathcal{K} \mathcal{A}}\left(X, \leq, \sim_{X}, Y_{X}, \mathcal{P}(X)\right) \in \mathbb{I S}\left(\mathbf{F}_{\mathcal{K} \mathcal{A}}\right)$.

Proof. Observe first that for each $x, y \in{\underset{\sim}{K}}^{n}, x \sim_{K^{n}} y$ iff there exists $z \leq x, y$. On the other hand, from the definition of Kleene spaces, it follows that for each Kleene space $\left(Z, \leq_{Z}, \sim_{Z}, Y_{Z}, \tau_{Z}\right)$, if $x, y, z \in Z$ are such that $z \leq_{Z} x, y$, then $x \sim_{Z} y$. This proves that any monotone map from $K_{\sim}^{n}$ into a Kleene space $\left(Z, \leq_{Z}, \sim_{Z}, Y_{Z}, \tau_{Z}\right)$ is a morphism in the category of Kleene spaces iff it sends $\{0,1\}^{n}$ into $Y_{Z}$.

We show that there exist $n \in\{1,2, \ldots\}$ and a monotone onto map $\eta: K_{\sim}^{n} \rightarrow\left(X, \leq, \sim_{X}\right.$ $\left., Y_{X}, \mathcal{P}(X)\right)$ such that $\eta\left(\{0,1\}^{n}\right)=Y_{X}=\min (X, \leq)$.

For $n=1,2, \ldots$, let $\left(B T(n), \leq_{n}\right)$ denote the perfect binary tree of depth $n$ (i.e., a tree in which every non-leaf node has exactly 2 children and all leaves are at depth $n$ ). Then for some $n \in\{1,2, \ldots\}$, there is an onto monotone map $\eta_{1}: B T(n) \rightarrow(X, \leq)$ such that $\eta_{1}\left(\min \left(B T(n), \leq_{n}\right.\right.$ )$)=\min (X, \leq)$. Consider $\sim_{B T(n)}=\leq_{n} \cup \geq_{n}$ and $Y_{B T(n)}=\min \left(B T(n), \leq_{n}\right)$. Then $\left(B T(n), \leq_{n}\right.$ $\left., \sim_{B T(n)}, Y_{B T(n)}, \mathcal{P}(B T(n))\right)$ is a Kleene space. It follows that $\eta_{1}$ is a morphism of Kleene spaces from $\left(B T(n), \leq_{n}, \sim_{B T(n)}, Y_{B T(n)}, \mathcal{P}(B T(n))\right)$ onto $\left(X, \leq_{,} \sim_{X}, Y_{X}, \mathcal{P}(X)\right)$.

Let $\eta_{2}: K_{\sim}^{n} \rightarrow K_{\sim}^{n}$ be defined as follows:

$$
\eta_{2}\left(x_{1}, \ldots, x_{n}\right)= \begin{cases}(a, \ldots, a) & \text { if } x_{1}=a \\ \left(x_{1}, \ldots, x_{n}\right) & \text { if } x_{j} \neq a \text { for } j=1 \ldots n \\ \left(x_{1}, \ldots, x_{i-1}, a, \ldots, a\right) & \text { if } i=\min \left\{j \in\{1, \ldots, n\} \mid x_{j}=a\right\} .\end{cases}
$$

Then $\eta_{2}$ is monotone and acts as the identity on $\{0,1\}^{n}$, and is hence a morphism of Kleene spaces.

By induction on $n$ it is easy to prove that $\eta_{2}\left(K_{\sim}^{n}\right)$ is a perfect binary tree of depth $n$. Therefore, considering it as a subobject of $K_{\sim}^{n}$, there is an isomorphism of Kleene spaces $\eta^{\prime}$ from $\eta_{2}\left(K_{\sim}^{n}\right)$ onto $\left(B T(n), \leq_{n}, \sim_{B T(n)}, Y_{B T(n)}, \mathcal{P}(B T(n))\right)$.

Finally, $\eta=\eta_{1} \circ \eta^{\prime} \circ \eta_{2}$ is a morphism of Kleene spaces from ${\underset{\sim}{n}}^{n}$ onto $\left(X, \leq, \sim_{X}, Y_{X}, \mathcal{P}(X)\right)$. By Theorem 31, $\mathrm{E}_{\mathcal{K} \mathcal{A}}(\eta): \mathrm{E}_{\mathcal{K} \mathcal{A}}\left(X, \leq, \sim_{X}, Y_{X}, \mathcal{P}(X)\right) \rightarrow \mathrm{E}_{\mathcal{K A} \mathcal{A}}\left(K^{n}\right)$ is a one-to-one homomorphism; that is, $\mathrm{E}_{\mathcal{K} \mathcal{A}}\left(X, \leq, \sim_{X}, Y_{X}, \mathcal{P}(X)\right) \in \mathbb{I S}\left(\mathbf{F}_{\mathcal{K} \mathcal{A}}(n)\right) \subseteq \mathbb{I S}\left(\mathbf{F}_{\mathcal{K} \mathcal{A}}\right)$, as required.

The algebra $\mathrm{E}_{\mathcal{K} \mathcal{A}}\left(X, \leq, \sim_{X}, Y_{X}, \mathcal{P}(X)\right)$ defined in this lemma not only embeds into $\mathbf{F}_{\mathcal{K} \mathcal{A}}$, it is also a retract of $\mathbf{F}_{\mathcal{K} \mathcal{A}}$. A characterization of finite retracts of free Kleene algebras, the finite projective algebras of this variety, is given in [38]. 


\subsection{Admissibility in Kleene Algebras and Lattices}

We obtain bases for admissibility in Kleene algebras and lattices following a similar procedure to that described in Section 5. First, we describe two properties of the dual space of finite nontrivial Kleene algebras using clauses.

Lemma 33. For a finite non-trivial Kleene algebra $\mathbf{A}$ with dual space $\mathbf{D}_{\mathcal{K} \mathcal{A}}(\mathbf{A})=(X, \leq, \sim, Y, \tau)$ :

(a) A satisfies (1) iff $X \neq \emptyset$.

(b) A satisfies (1) and (3) iff $(X, \leq)$ has a top element.

(c) A satisfies (1) and (8) iff $Y=\min (X, \leq) \neq \emptyset$.

Proof. (a) Straightforward, since A satisfies (1) iff it is non-trivial.

(b) Very similar to the argument used in the proof of Lemma 22 (ii) $\Rightarrow$ (iii).

(c) Assume for a contradiction that $\mathbf{A}$ satisfies (1) and (8) and $Y \subsetneq \min (X, \leq)$, and consider $y \in Y \backslash \min (X, \leq)$. We define $\eta, \mu: \mathrm{D}_{\mathcal{K} \mathcal{A}}(\mathbf{A}) \rightarrow \underset{\sim}{K}$ as follows:

$$
\eta(x)=\left\{\begin{array}{ll}
a & \text { if } x \geq y \\
1 & \text { otherwise }
\end{array} \quad \mu(x)= \begin{cases}a & \text { if } x \geq y \text { and } x \neq y \\
0 & \text { if } x=y \\
1 & \text { otherwise }\end{cases}\right.
$$

Then $\eta, \mu \in \mathrm{E}_{\mathcal{K} \mathcal{A}}\left(\mathrm{D}_{\mathcal{K} \mathcal{A}}(\mathbf{A})\right), \neg \eta \leq \eta, \eta \wedge \neg \mu=\neg \eta \leq \neg \eta \vee \mu$, and $\neg \mu \not \leq \mu$. Let $c=e_{\mathbf{A}}^{-1}(\eta)$ and $b=e_{\mathbf{A}}^{-1}(\mu)$. Then $\neg c \leq c, c \wedge \neg d \leq \neg c \vee d$, and $\neg d \not \leq d$, contradicting the assumption that $\mathbf{A}$ satisfies (8).

Conversely, assume that $Y=\min (X, \leq) \neq \emptyset$ and let $c, d \in A$ be such that $\neg c \leq c$ and $c \wedge \neg d \leq \neg c \vee d$. Then $\neg c \leq c$ implies $e_{\mathbf{A}}(c)(x) \in\{a, 1\}$ for each $x \in \mathrm{D}_{\mathcal{K}_{\mathcal{A}}}(\mathbf{A})$. Since $Y=\min (X, \leq)$, it follows that $e_{\mathbf{A}}(c)(y)=1$ for each $y \in \min (X, \leq)$. If there exists $x \in \mathrm{D}_{\mathcal{K} \mathcal{A}}(\mathbf{A})$ such that $e_{\mathbf{A}}(d)(x)=0$, then there exists $y \in \min (X, \leq)$ such that $e_{\mathbf{A}}(d)(y)=0$. It then follows that:

$$
\begin{aligned}
e_{\mathbf{A}}(c)(y) & =e_{\mathbf{A}}(c)(y) \wedge 1 \\
& =e_{\mathbf{A}}(c)(y) \wedge e_{\mathbf{A}}(\neg d)(y) \\
& \leq e_{\mathbf{A}}(\neg c)(y) \vee e_{\mathbf{A}}(d)(y) \\
& =e_{\mathbf{A}}(\neg c)(y) \vee 0 \\
& =e_{\mathbf{A}}(\neg c)(y) .
\end{aligned}
$$

This contradicts $e_{\mathbf{A}}(c)(y)=1$. So $e_{\mathbf{A}}(d)(x) \in\{a, 1\}$ for each $x \in \mathrm{D}_{\mathcal{K}_{\mathcal{A}}}(\mathbf{A})$, which implies that $\neg e_{\mathbf{A}}(d)(x) \leq e_{\mathbf{A}}(d)(x)$ and hence $\neg d \leq d$.

Lemma 34. Let $\mathbf{A}$ be a finite Kleene algebra and let $\mathrm{D}_{\mathcal{K} \mathcal{A}}(\mathbf{A})=(X, \leq, \sim, Y, \tau)$ be its dual space. Then the following are equivalent:

(i) $\mathbf{A} \in \mathbb{I S}\left(\mathbf{F}_{\mathcal{K} \mathcal{A}}\right)$.

(ii) $(X, \leq)$ has a top element and $Y=\min (X, \leq)$.

(iii) A satisfies (1), (3), and (8).

Proof. (ii) $\Leftrightarrow$ (iii). Immediate from Lemma 33 .

(i) $\Rightarrow$ (ii). By Theorems 1 and $31, \mathrm{D}_{\mathcal{K} \mathcal{A}}\left(\mathbf{F}_{\mathcal{K A}}(n)\right) \cong \widetilde{\sim}^{n}$. The result follows straightforwardly from the fact that $(a, \ldots, a) \in K^{n}$ is the top element of ${\underset{\sim}{N}}^{n}$, and that $\{0,1\}^{n}=\min \left(K_{\sim}^{n}\right)$.

(ii) $\Rightarrow$ (i). Let $M C$ be the set of maximal chains of $(X, \leq)$ and let $Z=\{(c, C) \in X \times M C \mid$ $c \in C\} \cup\{x\}$ where $x \notin X \times M C$. Let $\leq_{Z}$ be the partial order on $Z$ defined as follows:

$$
u \leq_{Z} v \quad \Leftrightarrow \quad v=x \quad \text { or } \quad u=\left(c_{1}, C\right), v=\left(c_{2}, C\right) \text {, and } c_{1} \leq c_{2} .
$$

Clearly, $\left(Z, \leq_{Z}\right)$ is a tree. Defining $Y_{Z}=\min \left(Z, \leq_{Z}\right)$ and $\sim_{Z}=\leq_{Z} \cup \geq_{Z}$, it follows from Lemma 32 that $\mathbf{B}=\mathrm{E}_{\mathcal{K} \mathcal{A}}\left(Z, \leq_{Z}, \sim_{Z}, Y_{Z}, \mathcal{P}(Z)\right)$ belongs to $\mathbb{I S}\left(\mathbf{F}_{\mathcal{K A}}\right)$. 
Let $\eta: Z \rightarrow X$ be the map defined by $\eta(c, C)=c$ and let $\eta(x)$ be the top element of $(X, \leq)$. From the definition of $\leq_{Z}, \eta$ is order-preserving, and, since $Y=\min (X, \leq), \eta$ maps $Y_{Z}$ into $Y$. It also follows that $\eta$ is onto $X$ and $\eta\left(\sim_{Z}\right)=\eta\left(\leq_{Z} \cup \geq_{Z}\right) \subseteq(\leq \cup \geq) \subseteq \sim$. Hence $\eta$ is a morphism of Kleene spaces from $\left(Z, \leq_{Z}, \sim_{Z}, Y_{Z}, \mathcal{P}(Z)\right)$ onto $(X, \leq, \sim, Y, \tau)$. The map $\mathrm{E}_{\mathcal{K} \mathcal{A}}(\eta) \circ e_{\mathbf{A}}: \mathbf{A} \rightarrow \mathbf{B}$ is therefore a one-to-one homomorphism. Hence $\mathbf{A} \in \mathbb{I S}(\mathbf{B}) \subseteq \mathbb{I} S\left(\mathbf{F}_{\mathcal{K A}}\right)$.

So now by Lemmas 12,33 , and 34 :

Theorem 35. $\{(1),(3),(8)\}$ is a basis for the admissible clauses of Kleene algebras.

Turning our attention next to quasi-identities:

Lemma 36. Let $\mathbf{A}$ be a finite Kleene algebra and let $\mathrm{D}_{\mathcal{K A}}(\mathbf{A})=(X, \leq, \sim, Y, \tau)$ be its dual space. Then the following are equivalent:

(i) $\mathbf{A} \in \mathbb{I S P}\left(\mathbf{F}_{\mathcal{K} \mathcal{A}}\right)$.

(ii) $Y=\min (X, \leq)$.

(iii) A satisfies (8).

Proof. (ii) $\Leftrightarrow$ (iii). The equivalence follows using Lemma 33 parts (a) and (c), and the observation that $Y=\min (X, \leq)=\emptyset$ implies $X=\emptyset$.

(i) $\Rightarrow$ (iii). $\mathcal{K} \mathcal{A}$ is locally finite, so every finitely generated subalgebra of $\mathbf{F}_{\mathcal{K} \mathcal{A}}$ is finite and, by Lemma 34, satisfies (8). It follows that $\mathbf{F}_{\mathcal{K} \mathcal{A}}$ and hence every algebra in $\operatorname{ISP}\left(\mathbf{F}_{\mathcal{K} \mathcal{A}}\right)$ satisfies this quasi-identity.

(ii) $\Rightarrow$ (i). Follows by a similar argument to that given in the proof of Lemma 26 (ii) $\Rightarrow$ (i).

Hence, by Lemmas 12 and 36, we obtain (by different means) the result of [21]:

Theorem 37. $\{(8)\}$ is a basis for the admissible quasi-identities of Kleene algebras.

Finally, we provide bases for the admissible clauses and admissible quasi-identities of the class of Kleene lattices.

Lemma 38. Let $\mathbf{A}$ be a finite Kleene lattice. Then the following are equivalent:

(i) $\mathbf{A} \in \mathbb{I S}\left(\mathbf{F}_{\mathcal{K} \mathcal{L}}\right)$.

(ii) A satisfies (4) and (8).

Proof. Observe first that $\mathbf{A} \in \mathbb{I S}\left(\mathbf{F}_{\mathcal{K} \mathcal{L}}\right)$ iff $\overline{\mathbf{A}} \in \mathbb{I S}\left(\mathbf{F}_{\mathcal{K} \mathcal{A}}\right)$. Hence, by Lemma 34 , $\mathbf{A} \in \mathbb{I S}\left(\mathbf{F}_{\mathcal{K} \mathcal{L}}\right)$ iff $\overline{\mathbf{A}}$ satisfies (1), (3), and (8). Since $\overline{\mathbf{A}}$ always satisfies (1) and (3), it suffices to prove that $\overline{\mathbf{A}}$ satisfies (8) iff A satisfies (4) and (8).

Suppose that $\overline{\mathbf{A}}$ satisfies (8). Then clearly A satisfies (8). Also, if $c \in \mathbf{A}$ satisfies $c=\neg c$, then $c=c \wedge \neg \perp \leq \neg c \vee \perp=c$ in $\overline{\mathbf{A}}$ and, by (8), it follows that $T=\neg \perp \leq \perp$, a contradiction. Hence A satisfies (4).

For the converse, suppose that $\mathbf{A}$ satisfies (4) and (8). Consider $c, d \in \overline{\mathbf{A}}$ such that $\neg c \leq c$ and $c \wedge \neg d \leq \neg c \vee d$. Observe that $d \neq \perp$. Indeed, if $d=\perp$, then $c=c \wedge \top \leq \neg c \vee \perp=\neg c$, so $c=\neg c$. But then $c \notin\{\perp, \top\}$ and, as $\mathbf{A}$ satisfies (4), also $c \notin A$, a contradiction. The following cases remain: (i) If $c, d \in A$, then, as $\mathbf{A}$ satisfies (8), also $\neg d \leq d$; (ii) If $c \in\{\perp, \top\}$, then, as $\neg c \leq c$, it follows that $c=\top$ and $\neg d=\top \wedge \neg d \leq \perp \vee d=d$; (iii) If $d=\top$, clearly $\neg d=\perp \leq \top=d$.

Lemma 39. Let $\mathbf{A}$ be a finite Kleene lattice. Then the following are equivalent:

(i) $\mathbf{A} \in \mathbb{I S P}\left(\mathbf{F}_{\mathcal{K L}}\right)$

(ii) A satisfies (8). 
Proof. (i) $\Rightarrow$ (ii). $\mathcal{K} \mathcal{L}$ is locally finite, so every finitely generated subalgebra of $\mathbf{F}_{\mathcal{K L}}$ is finite and, by Lemma 38, satisfies (8). It follows that $\mathbf{F}_{\mathcal{K} \mathcal{L}}$ and hence every algebra in $\operatorname{ISP}\left(\mathbf{F}_{\mathcal{K} \mathcal{L}}\right)$ satisfies (8).

(ii) $\Rightarrow$ (i). If $\mathbf{A}$ satisfies also (4), then by Lemma 38, $\mathbf{A} \in \mathbb{I S}\left(\mathbf{F}_{\mathcal{K L}}\right) \subseteq \mathbb{I S P}\left(\mathbf{F}_{\mathcal{K} \mathcal{L}}\right)$. If $\mathbf{A}$ does not satisfy (4), there exists $c \in \mathbf{A}$ such that $\neg c=c$. Then for any $d \in \mathbf{A}$,

$$
c \wedge \neg(c \wedge d) \leq c=\neg c \leq \neg c \vee(c \wedge d) .
$$

By (8), it follows that $\neg(c \wedge d) \leq c \wedge d$. On the other hand, as $c \wedge d \leq c$,

$$
c \wedge d \leq c=\neg c \leq \neg(c \wedge d) .
$$

It follows that $c \wedge d=\neg(c \wedge d)=c$. So $\mathbf{A}$ is a trivial algebra and a member of $\operatorname{ISP}\left(\mathbf{F}_{\mathcal{K} L}\right)$.

Hence, by Lemmas 12, 38, and 39:

Theorem 40. $\{(4),(8)\}$ and $\{(8)\}$ are bases for the admissible clauses and admissible quasiidentities of Kleene lattices, respectively.

\section{Acknowledgements}

The authors acknowledge support from Marie Curie Reintegration grant PIRG06-GA-2009-256492 and Swiss National Science Foundation grants 20002_129507 and grant 200021_146748. We thank Christoph Röthlisberger for his helpful comments, and are grateful also to two anonymous referees who noted several inconsistencies in the original version of this paper and suggested many valuable improvements.

[1] P. Lorenzen, Einführung in die operative Logik und Mathematik, volume 78 of Grundlehren der mathematischen Wissenschaften, Springer, 1955.

[2] G. Gentzen, Untersuchungen über das Logische Schliessen, Mathematische Zeitschrift 39 (1935) $176,-210,405-431$.

[3] I. Johansson, Der minimalkalkul, ein reduzierter intuitionistischer formalismus, Compositio Mathematica 4 (1936) 119-136.

[4] P. Whitman, Free lattices, Annals of Mathematics 42 (1941) 325-329.

[5] G. Metcalfe, N. Olivetti, D. Gabbay, Proof Theory for Fuzzy Logics, Springer, 2008.

[6] G. Metcalfe, F. Montagna, Substructural fuzzy logics, Journal of Symbolic Logic 72 (2007) 834-864.

[7] V. Rybakov, A criterion for admissibility of rules in the modal system $\mathrm{S} 4$ and the intuitionistic logic, Algebra Logic 23 (1984) 369-384.

[8] V. Rybakov, Admissibility of Logical Inference Rules, volume 136 of Studies in Logic and the Foundations of Mathematics, Elsevier, Amsterdam, 1997.

[9] R. Iemhoff, On the admissible rules of intuitionistic propositional logic, Journal of Symbolic Logic 66 (2001) 281-294.

[10] P. Rozière, Regles Admissibles en calcul propositionnel intuitionniste, Ph.D. thesis, Université Paris VII, 1992.

[11] R. Iemhoff, Intermediate logics and Visser's rules, Notre Dame Journal of Formal Logic 46 (2005) 65-81.

[12] P. Cintula, G. Metcalfe, Admissible rules in the implication-negation fragment of intuitionistic logic, Annals of Pure and Applied Logic 162 (2010) 162-171. 
[13] E. Jeřábek, Admissible rules of modal logics, Journal of Logic and Computation 15 (2005) 411-431.

[14] S. Babenyshev, V. Rybakov, Unification in linear temporal logic LTL, Annals of Pure and Applied Logic 162 (2011) 991-1000.

[15] S. Babenyshev, V. Rybakov, Linear temporal logic LTL: Basis for admissible rules, Journal of Logic and Computation 21 (2011) 157-177.

[16] E. Jeřábek, Admissible rules of Łukasiewicz logic, Journal of Logic and Computation 20 (2010) 425-447.

[17] E. Jeřábek, Bases of admissible rules of Łukasiewicz logic, Journal of Logic and Computation 20 (2010) 1149-1163.

[18] S. Ghilardi, Unification in intuitionistic logic, Journal of Symbolic Logic 64 (1999) 859-880.

[19] S. Ghilardi, Best solving modal equations, Annals of Pure and Applied Logic 102 (2000) 184-198.

[20] D. M. Clark, B. A. Davey, Natural Dualities for the Working Algebraist, Cambridge University Press, 1998.

[21] G. Metcalfe, C. Röthlisberger, Admissibility in De Morgan algebras, Soft Computing 16 (2012) 1875-1882.

[22] G. Metcalfe, C. Röthlisberger, Admissibility in finitely generated quasivarieties, Logical Methods in Computer Science 9 (2013) 1-19.

[23] N. Belnap, How a computer should think, in: G. Ryle (Ed.), Contemporary Aspects of Philosophy, Oriel Press Ltd., 1977, pp. 30-56.

[24] M. Gehrke, C. Walker, E. Walker, Fuzzy logics arising from strict De Morgan systems, in: Topological and Algebraic Structures in Fuzzy Sets, volume 20 of Trends in Logic, pp. $257-276$.

[25] S. M. Lane, Categories for the Working Mathematician, volume 5 of Graduate Texts in Mathematics, Springer, 1971.

[26] S. Burris, H. P. Sankappanavar, A Course in Universal Algebra, volume 78 of Graduate Texts in Mathematics, Springer-Verlag, New York, 1981.

[27] C. Chang, H. Keisler, Model Theory, volume 73 of Studies in Logic and the Foundations of Mathematics, Elsevier, 1977.

[28] B. Jónsson, Sublattices of a free lattice, Canadian Journal of Mathematics 13 (1961) 256-264.

[29] L. Fuchs, Abelian Groups, Pergamon Press, 1960.

[30] C. Bergman, Structural completeness in algebra and logic, in: H. Andréka, J. Monk, I. Nemeti (Eds.), Algebraic Logic, volume 54 of Colloquia Mathematica Societatis János Bolyai, NorthHolland, Amsterdam, 1991, pp. 59-73.

[31] W. M. Beynon, Applications of duality in the theory of finitely generated lattice-ordered abelian groups, Canadian Journal of Mathematics 29 (1977) 243-254.

[32] H. A. Priestley, Representation of distributive lattices by means of ordered Stone spaces, Bulletin of the London Mathematical Society 2 (1970) 186-190.

[33] R. Balbes, A. Horn, Projective distributive lattices, Pacific Journal of Mathematics 33 (1970) $273-279$. 
[34] G. Grätzer, General Lattice Theory, Birkhäuser, Basel, second edition, 1998.

[35] B. A. Davey, Dualities for Stone algebras, double Stone algebras and relative Stone algebras, Colloquia Mathematica Societatis János Bolyai 46 (1982) 1-14.

[36] A. P. Pynko, Implicational classes of De Morgan lattices, Discrete Mathematics 205 (1999) $171-181$.

[37] J. A. Kalman, Lattices with involution, Transactions of the American Mathematical Society 87 (1958) 485-491.

[38] S. Bova, L. M. Cabrer, Unification and projectivity in De Morgan and Kleene algebras, Order 31 (2014) 159-187.

[39] B. A. Davey, H. Werner, Piggyback-dualities, Bulletin of the Australian Mathematical Society $32(1985) 1-32$. 